By

\author{
Jacob Rubinstein \\ Peter Sternberg \\ and
}

Gershon Wolansky

IMA Preprint Series \# 2012

(December 2004)

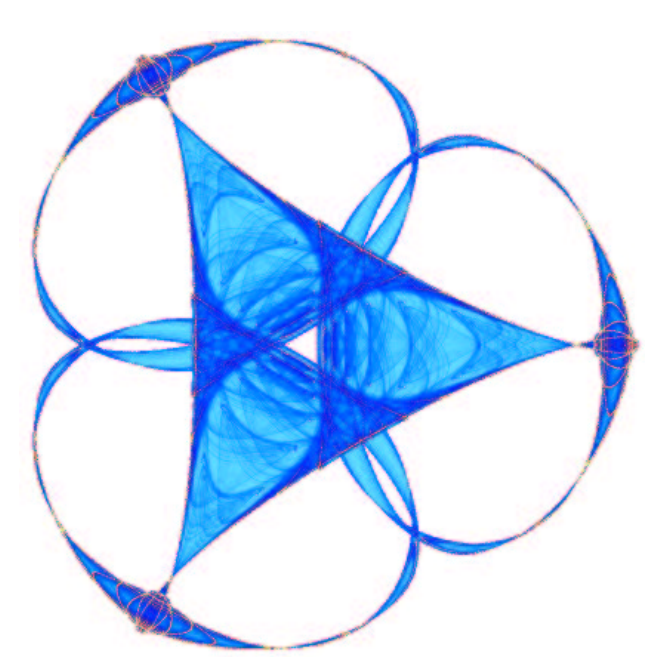

INSTITUTE FOR MATHEMATICS AND ITS APPLICATIONS

UNIVERSITY OF MINNESOTA

514 Vincent Hall

206 Church Street S.E.

Minneapolis, Minnesota 55455-0436

Phone: 612/624-6066 Fax: 612/626-7370

URL: http://www.ima.umn.edu 


\title{
Elliptic problems on networks with constrictions
}

\author{
Jacob Rubinstein ${ }^{1}$ \\ Department of Mathematics \\ Indiana University \\ Bloomington, IN 47405 \\ Peter Sternberg ${ }^{2}$ \\ Department of Mathematics \\ Indiana University \\ Bloomington, IN \\ 47405 \\ Gershon Wolansky ${ }^{3}$ \\ Department of Mathematics \\ Technion, I.I.T. \\ 32000 Haifa, Israel
}

Key Words: Differential equations on graphs, Ginzburg-Landau theory AMS Subject Classification: 35Q60, 78M30, 78M35.

\begin{abstract}
We investigate the asymptotic behavior of minimizers to sequences of elliptic variational problems posed on thin three-dimensional domains. These domains arise as thin neighborhoods of artibrary graphs that contain severe constrictions near the graph nodes. We characterize an appropriate limit of minimizers as a function of one variable defined on the graph that necessarily minimizes a one-dimensional variational problem. The most salient feature of these limits of minimizers is the emergence of jump discontinuities across the graph nodes. While the approach can handle quite general elliptic problems, we pay particular attention to an application to generalized Josephson junctions within the Ginzburg-Landau theory of superconductivity.
\end{abstract}

\footnotetext{
${ }^{1}$ Research partially supported by NSF DMS-0203312. email: jrubinst@indiana.edu

${ }^{2}$ Research partially supported by NSF DMS-0100540. email: sternber@indiana.edu

${ }^{3}$ Research partially supported by Israel Science Foundation grant no.77/01. email: gershonw@math.technion.ac.il
} 


\section{Introduction}

This paper concerns asymptotic limits for elliptic variational problems in networks with constrictions. By a constricted network we mean a threedimensional domain representing a thin neighborhood of a three-dimensional graph. This graph may consist of a finite number of smooth curves joined at a finite number of nodes and the three-dimensional domain is taken to be particularly thin in a neighborhood of each of the nodes. In the limit where the thickness of the domain shrinks to zero and the domain collapses to the graph, we characterize the asymptotic behavior of minimizers by identifying a limiting variational problem defined on the graph in the spirit of Gammaconvergence. We then argue that this limiting functional is minimized by a limit of minimizers of the finite thickness problems.

Elliptic problems posed on constricted domains arise in a number of settings. One can for example find in [4] a study of the elasticity of notched beams. Another setting is the study of diffusion processes taking place over a collection of thin constricted pipes. One of our primary motivations lies in applications to the Ginzburg-Landau theory of superconductivity. For the case of superconductivity, we have in mind the modeling of a weak link in a superconducting wire, cf. [12], [1]. This is a kind of "geometrical" Josephson junction [9] in which a supercurrent successfully tunnels across a very narrow portion of a wire. Such constrictions are of interest because they are known to give rise to currents which are proportional to the sine of the phase jump across the constriction. When more than one constriction is present in a looped wire, interference patterns can develop leading to interesting relationships between the supercurrent and the magnetic flux through the loop. The most famous device exploiting this phenomenon is the SQUID, used to measure tiny fluctuations in magnetic flux. In [15] we initiated this kind of investigation for the case of a three-dimensional torus with a single constriction. Here we develop an asymptotic theory of elliptic variational problems posed on constricted networks that accomodates arbitrary geometries and multiple incoming branches at each constriction.

Before describing our results as they apply to Ginzburg-Landau, we first explain them in the simplest possible setting, that of minimizing the Dirichlet integral

$$
\inf _{\mathcal{A}_{\varepsilon}} \int_{\Omega_{\varepsilon}}|\nabla u|^{2} d x
$$

over an admissible set $\mathcal{A}_{\varepsilon}$ consisting of functions with square-integrable gra- 
dients which additionally satisfy some inhomogeneous boundary condition such as Dirichlet on part of the domain so as to keep the minimizer $u^{\varepsilon}$ from being constant. Here $\Omega_{\varepsilon}$ is taken to be a thickened graph consisting of $N$ smooth curves in $\mathbb{R}^{3}$ sharing the origin as an endpoint and a constant Dirichlet condition is applied at the end of each cylindrical thickening of the graph. (See Figure 1 for a depiction with the curves taken to be line segments.) We first take appropriately scaled integral averages of the $u^{\varepsilon}$ taken over crosssections of the cylinders and prove a compactness result that establishes a subsequential limit $U^{0}$ defined on the limiting graph $\Gamma$, cf. Proposition 2.4. Then we show in Theorem 2.5 that $U^{0}$ minimizes the limiting energy

$$
\inf _{U \in \mathcal{A}_{\Gamma}, \alpha \in \mathbb{R}^{1}}\left\{\sum_{k=1}^{N} \int_{\Gamma_{k}}\left(U_{k}^{\prime}\right)^{2} d s+b \sum_{k=1}^{N}\left|U_{k}(0)-\alpha\right|^{2}\right\},
$$

where $U_{k}$ is the restriction of a function $U \in H^{1}(\Gamma \backslash\{0\})$ to the $k^{\text {th }}$ branch $\Gamma_{k}$ of the graph and $U_{k}(0)$ denotes its limiting value as one approaches the origin along $\Gamma_{k}$. The admissible set $\mathcal{A}_{\Gamma}$ is given by

$$
\mathcal{A}_{\Gamma}:=\left\{U \in H^{1}(\Gamma \backslash\{0\}): U_{k}\left(L_{k}\right)=c_{k} \text { for } k=1, \ldots, N\right\},
$$

where $L_{k}$ denotes the length of $\Gamma_{k}$ and the constants $c_{1} \ldots, c_{N}$ denote the $N$ Dirichlet conditions referred to above. The constant $b>0$ is a factor related to the geometry of the constriction.

By proving that a limit of the sequence $\left\{u^{\varepsilon}\right\}$ minimizes (1.2), we conclude that in particular, a limit of minimizers is discontinuous at the origin. Note that minimization of (1.2) over $\alpha$ leads easily to the fact that the optimal $\alpha$ is simply the average of the numbers $U_{1}(0), \ldots U_{N}(0)$, but in general these $N$ numbers will be distinct. Heuristically speaking, we are thus finding that minimizers of (1.1) will undergo a relatively inexpensive, rapid transition across the constriction, with a jump discontinuity emerging in the limit. This behavior is in stark contrast to the kind of results found in [10] and [11], where less severe (2-d) constrictions, or even swellings are considered at nodes of a graph and the limit of minimizers is characterized as being continuous at the junctions.

Regarding the application of our techniques to Ginzburg-Landau theory, we develop much further here the approach initiated in [15]. In [15], we already demonstrated that an asymptotic analysis of the Ginzburg-Landau energy (for a definition, see (3.1)) in the presence of a constriction can lead to a sinusoidal Josephson condition mentioned earlier in the introduction. This 
investigation considered a circular torus with one constriction which collapses to a circle in the zero thickness limit. This torus was subjected to an applied magnetic field directed orthogonal to the circle. In the present article, we wish to consider arbitrary applied magnetic fields and to replace the circle with a consideration of arbitrary three-dimensional graphs, graphs which in particular may possess nodes joining an arbitrary number of curves (wires). As in the study of the harmonic minimizers of (1.1), we derive a compactness result for minimizers of the Ginzburg-Landau energy on constricted networks collapsing to a graph, and then show the limit of minimizers necessarily minimizes a limiting variatonal problem posed on the graph, cf. Theorem 3.6. Through consideration of the resulting natural boundary conditions at each node, this will allow us to derive a kind of generalized Josephson condition at each node involving a finite sum of sine functions. Suppose, for example, that for a particular node $P$ on the graph, there are $N$ curves $\Gamma_{1}, \ldots, \Gamma_{N}$ sharing the endpoint $P$. Let us denote the supercurrent directed along $\Gamma_{k}$ towards $P$ by $J_{k}$, and let $\phi_{1}, \ldots, \phi_{N}$ denote the phases of the complex order parameter minimizing the Ginzburg-Landau energy. Then through the natural boundary conditions satisfied by a minimizer of the limiting energy, we find the formula

$$
J_{k} \propto \sum_{l \neq k} \sin \left(\phi_{k}-\phi_{l}\right) \quad \text { for } k=1, \ldots, N
$$

In particular, for the case $N=2$ of two wires joined at a constriction, one recovers the standard Josephson condition

$$
J_{1} \propto \sin \left(\phi_{1}-\phi_{2}\right) \text { and } J_{2}=-J_{1} .
$$

The discontinuity of the minimizer to the 1-d limit contrasts with the continuity of minimizers obtained for Ginzburg-Landau under the collapse of domains with less severe constrictions in [13].

Having derived (1.3), and through a (formal) manipulation that allows us to relate phase jumps to magnetic flux through holes of a graph, our hope is to obtain current/flux relationships for arbitrarily complicated configurations of wires with weak links in order to perhaps suggest new and interesting superconducting devices. We pursue these ideas in [16].

In Section 2 we describe and prove our results for the simplest setting (1.1) for a domain $\Omega_{\varepsilon}$ collapsing to a finite set of line segments meeting at the origin. Then in Section 3 we extend the results to arbitrary graphs and to the Ginzburg-Landau setting. 
Acknowledgment. P.S. would like to thank the Institute for Mathematics and its Applications in Minneapolis for its hospitality during the writing of this article.

\section{Asymptotic behavior of harmonic functions in multiply constricted domains}

We begin with a description of the geometry of the region $\Omega_{\varepsilon}$ to be considered. To this end, we begin with a graph in $\mathbb{R}^{3}$ consisting of a union of $N$ line segments $\Gamma_{k}$ of length $L_{k}, k=1, \ldots, N$, all having one endpoint in common, located at the origin of a Cartesian coordinate system with points denoted by $x=\left(x_{1}, x_{2}, x_{3}\right)$. It will be convenient to express each line segment $\Gamma_{k}$ parametrically as the image of the interval $0 \leq s \leq L_{k}$ under the map $\gamma_{k}: \mathbb{R}^{1} \rightarrow \mathbb{R}^{3}$ given by $\gamma_{i}(s)=\gamma_{i}^{\prime}(0) s$ for some constant $\gamma_{k}^{\prime}(0) \in \mathbb{R}^{3}$.

The domain under consideration will be a certain neighborhood of this graph and to describe it precisely, we also introduce for every positive $\varepsilon$, a piecewise linear function $g_{\varepsilon}:[0, \infty) \rightarrow \mathbb{R}^{1}$ that will govern the thickness of the $N$ branches of our domain. Fixing any numbers $p \in(0,1)$ and $b>0$, we define

$$
g^{\varepsilon}(s):=\left\{\begin{array}{cc}
b \varepsilon^{1+p} & \text { for } \quad 0 \leq s \leq \varepsilon^{1+p} \\
\text { linear } & \text { for } \quad \varepsilon^{1+p}<s<\varepsilon^{p} \\
\varepsilon & \text { for } \quad \varepsilon^{p} \leq s<\infty
\end{array}\right.
$$

Now for each vector $\gamma_{k}^{\prime}(0)$ we select unit vectors $\mathbf{n}_{k}$ and $\mathbf{b}_{k}$ so that $\left(\gamma_{k}^{\prime}(0), \mathbf{n}_{k}, \mathbf{b}_{k}\right)$ forms an orthonormal basis for $\mathbb{R}^{3}$. The notation $\mathbf{n}_{k}$ and $\mathbf{b}_{k}$ is chosen so as to make for a smoother transition in the next section to a Frenet frame in the case of curved graphs. Then define the maps $T_{k}^{\varepsilon}: \mathbb{R}^{3} \rightarrow \mathbb{R}^{3}$ via the formula

$$
T_{k}^{\varepsilon}(s, y, z)=\gamma_{k}(s)+y g^{\varepsilon}(s) \mathbf{n}_{k}+z g^{\varepsilon}(s) \mathbf{b}_{k} .
$$

Using the notation $\mathcal{C}_{L}:=\left\{(s, y, z): 0 \leq s \leq L, y^{2}+z^{2}<1\right\}$ to denote the cylinder of length $L$, note that the set $C_{k}^{\varepsilon}:=T_{k}^{\varepsilon}\left(\mathcal{C}_{L_{k}}\right)$ consists of a tapered cylinder with central axis along $\Gamma_{k}$ of length $L_{k}$ and cross-sectional radius $g^{\varepsilon}(s)$. We can now define an $N$-branched region $\Omega_{\varepsilon} \subset \mathbb{R}^{3}$ as the union of the $N$ solid open cylinders with central axis along $\Gamma_{k}$ and radius $g^{\varepsilon}(s), s \in\left(0, L_{k}\right)$ and the open ball centered at the origin of radius $b \varepsilon^{1+p}$; that is

$$
\Omega_{\varepsilon}:=C_{1}^{\varepsilon} \cup \ldots \cup C_{N}^{\varepsilon} \cup B\left(0, b \varepsilon^{1+p}\right) .
$$




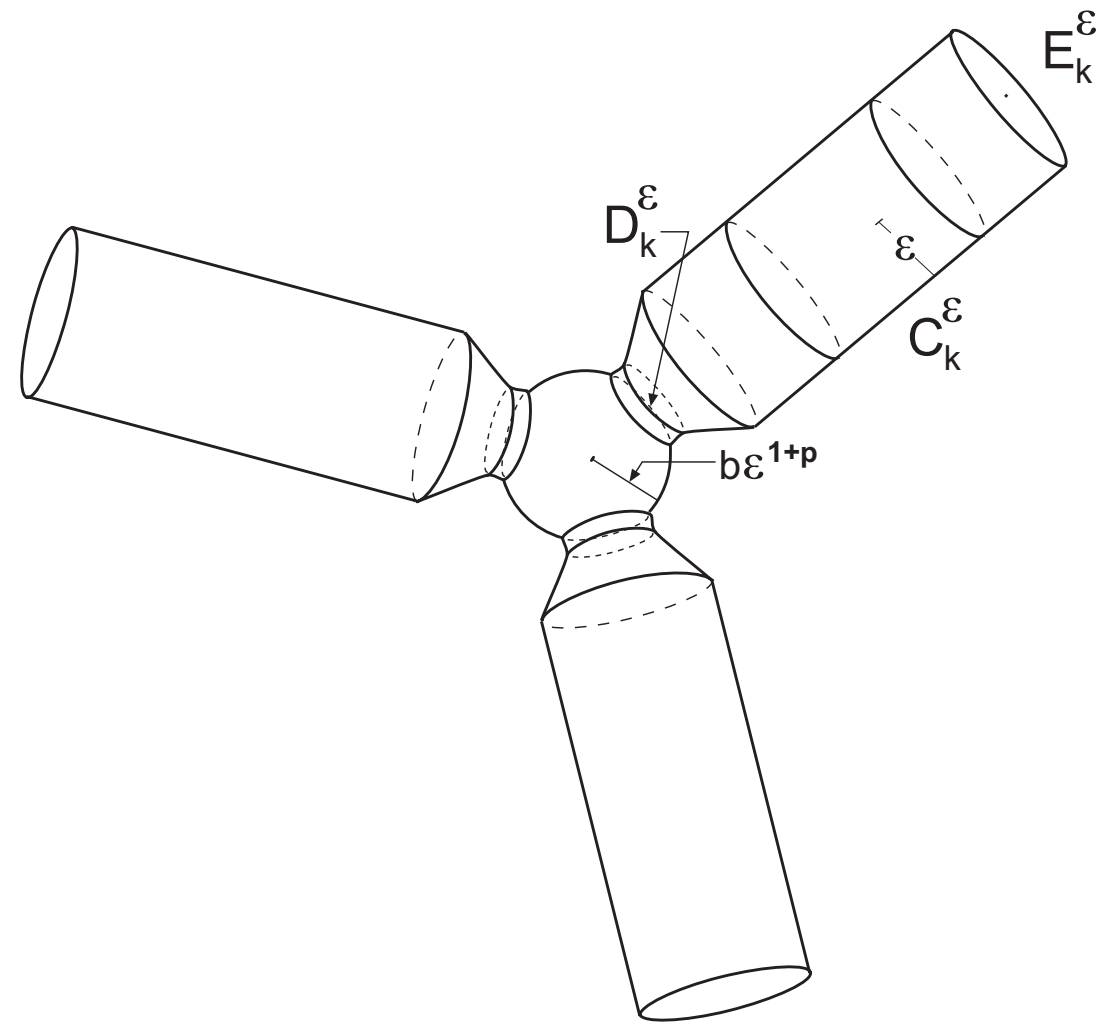

Figure 1: A domain $\Omega_{\varepsilon}$ consisting of multiple branches constricted at a node. Please note that the figure is not drawn to scale.

See Figure 1.

We devote this section to the model problem of determining the asymptotic behavior of the harmonic functions minimizing the Dirichlet integral taken over $\Omega_{\varepsilon}$, subject to specified inhomogeneous constant Dirichlet boundary conditions on the ends of the branches. We wish to emphasize that this example has been chosen in order to exhibit the result through perhaps the simplest possible setting yielding a nontrivial minimizer. The result holds for much more general elliptic operators and for other kinds of boundary conditions and the approach of this section can serve as a building block for these generalizations. In particular, a more complicated and more interesting example relating to superconductivity appears in the following section.

Let $E_{k}^{\varepsilon}, k=1,2, \ldots, N$ denote the discs of radius $\varepsilon$ forming the ends of the $N$ cylindrical branches, corresponding to $s=L_{1}, \ldots, L_{N}$ and let $c_{1}, \ldots, c_{N}$ denote any $N$ constants. We will pursue an asymptotic description of a sequence of minimizers to the variational problems

$$
\inf _{\mathcal{A}_{\varepsilon}} \int_{\Omega_{\varepsilon}}|\nabla u|^{2} d x,
$$


where

$$
\mathcal{A}_{\varepsilon}:=\left\{u \in H^{1}\left(\Omega_{\varepsilon}\right): u(x)=c_{k} \text { for } x \in E_{k}^{\varepsilon}, k=1, \ldots, N\right\} .
$$

By a standard application of the direct method, a minimizer $u^{\varepsilon}$ exists for each $\varepsilon>0$. This minimizer will clearly be a harmonic function in $\Omega_{\varepsilon}$, satisfying the Dirichlet condition on the discs $E_{k}^{\varepsilon}$ and satisfying the 'natural' homogeneous Neumann conditions on the remainder of $\partial \Omega_{\varepsilon}$. Furthermore, elementary use of the maximum principle implies that

$$
\left|u^{\varepsilon}(x)\right| \leq \max \left\{\left|c_{1}\right|, \ldots,\left|c_{N}\right|\right\} \text { for all } x \in \Omega_{\varepsilon} \text {. }
$$

Next we construct a sequence of simple test functions $\left\{v^{\varepsilon}\right\}$ on $\Omega_{\varepsilon}$ in order to bound the energy of $u^{\varepsilon}$ as follows. Along the $k^{t h}$ branch, define $v^{\varepsilon}$ to be a linear function of the variable running along $\Gamma_{k}$, taking the value 0 on the disc $D_{k}^{\varepsilon}:=T_{k}^{\varepsilon}\left(\left\{\left(\varepsilon^{1+p}, y, z\right): y^{2}+z^{2}<1\right\}\right.$ and $c_{k}$ along the disc $T_{k}^{\varepsilon}\left(\left\{L_{k}, y, z\right): y^{2}+z^{2}<1\right\}$, and then set $v^{\varepsilon} \equiv 0$ on the region

$$
\tilde{B}_{\varepsilon}:=\Omega_{\varepsilon} \backslash \bigcup_{k=1}^{N} T_{k}^{\varepsilon}\left(\left\{(s, y, z): \varepsilon^{1+p}<s<L_{k}, y^{2}+z^{2}<1\right\}\right) .
$$

One then readily checks that

$$
\int_{\Omega_{\varepsilon}}\left|\nabla u^{\varepsilon}\right|^{2} d x \leq \int_{\Omega_{\varepsilon}}\left|\nabla v^{\varepsilon}\right|^{2} d x \leq C \varepsilon^{2},
$$

since the volume of $\Omega_{\varepsilon}$ is $\mathcal{O}\left(\varepsilon^{2}\right)$.

We will describe the asymptotic behavior of the minimizers using the variables $(s, y, z)$. To this end, we now introduce $U_{k}^{\varepsilon}$ through the relation

$$
U_{k}^{\varepsilon}(s, y, z)=u^{\varepsilon}\left(T_{k}^{\varepsilon}(s, y, z)\right)
$$

In light of the estimate (2.6), it is apparent that one should rescale the energy by dividing by $\varepsilon^{2}$ in order to capture the leading order behavior in the $\varepsilon \rightarrow 0$ limit. With this in mind, for any numbers $\delta_{1}$ and $\delta_{2}$ satisfying $0 \leq \delta_{1}<\delta_{2} \leq L_{k}$, we use (2.2) to change variables and compute

$$
\begin{aligned}
& \frac{1}{\varepsilon^{2}} \int_{T_{k}^{\varepsilon}\left(\left\{(s, y, z): \delta_{1}<s<\delta_{2}, y^{2}+z^{2}<1\right\}\right)}\left|\nabla u^{\varepsilon}\right|^{2} d x= \\
& \int_{\delta_{1}}^{\delta_{2}} \int_{\left\{y^{2}+z^{2}<1\right\}}\left|\left(U_{k}^{\varepsilon}\right)_{s}-\frac{\left(g^{\varepsilon}\right)^{\prime}}{g^{\varepsilon}}\left(y\left(U_{k}^{\varepsilon}\right)_{y}+z\left(U_{k}^{\varepsilon}\right)_{z}\right)\right|^{2} \frac{g^{\varepsilon}(s)^{2}}{\varepsilon^{2}} d y d z d s \\
& +\frac{1}{\varepsilon^{2}} \int_{\delta_{1}}^{\delta_{2}} \int_{\left\{y^{2}+z^{2}<1\right\}}\left|\left(U_{k}^{\varepsilon}\right)_{y}\right|^{2}+\left|\left(U_{k}^{\varepsilon}\right)_{z}\right|^{2} d y d z d s,
\end{aligned}
$$


One immediate consequence of combining (2.6) and (2.8) is the estimate

$$
\int_{0}^{L_{k}} \int_{\left\{y^{2}+z^{2}<1\right\}}\left|\left(U_{k}^{\varepsilon}\right)_{y}\right|^{2}+\left|\left(U_{k}^{\varepsilon}\right)_{z}\right|^{2} d y d z d s \leq C \varepsilon^{2}
$$

which suggests (not surprisingly) that in the limit, what will matter is the $s$-dependence of the minimizers.

A crucial aspect of the approach is to gain some control on the asymptotic behavior of the minimizers $u^{\varepsilon}$ within $\tilde{B}_{\varepsilon}$. While the gradient of $u^{\varepsilon}$ is typically blowing up near the constriction, we see from the following proposition that the average of $u^{\varepsilon}$ over each of the discs $D_{k}^{\varepsilon}$ is under control:

2.1 Proposition. There exists a subsequence $\left\{\varepsilon_{j}\right\} \rightarrow 0$ and a number $\alpha_{0}$ such that

$$
\lim _{\varepsilon_{j} \rightarrow 0} f_{D_{k}^{\varepsilon_{j}}} u^{\varepsilon_{j}} d S=\alpha_{0} \quad \text { for } k=1, \ldots, N .
$$

2.2 Remark. Here and throughout, and notation $f_{S}$. denotes the integral average of a function over the domain of integration $S$, i.e. $\frac{1}{|S|} \int_{S} \cdot$

As we shall see, this proposition follows easily from the following elementary lemma.

2.3 Lemma. Let $\Omega \subset \mathbb{R}^{n}$ be a bounded domain with a smooth boundary. Let $D_{1}, \ldots D_{N} \subset \partial \Omega$ be mutually disjoint domains. Given constants $\left\{a_{k}\right\} \subset \mathbb{R}^{1}$, $1 \leq k \leq N$, define

$C_{\Omega}\left(a_{1}, \ldots a_{N}\right)=\inf _{u \in H^{1}(\Omega)}\left\{\int_{\Omega}|\nabla u|^{2} d x: f_{D_{k}} u d H^{n-1}(x)=a_{k}, k=1, \ldots, N\right\}$.

Then function $C_{\Omega}$ is a quadratic form on $\mathbb{R}^{N}$, namely

$$
C_{\Omega}\left(a_{1}, \ldots a_{N}\right)=\mathbf{a} \mathcal{C}_{B} \mathbf{a}^{t}
$$

where $\mathbf{a}=\left(a_{1}, \ldots a_{N}\right), \mathbf{a}^{t}$ denotes its transpose, and $\mathcal{C}_{\Omega}$ is an $N \times N$ symmetric, non-negative matrix satisfying

$$
\mathbf{a} \bigodot_{B} \mathbf{a}^{t}=0 \quad \text { iff } \mathbf{a}=\lambda(1, \ldots, 1) \quad \text { for some } \lambda \in \mathbb{R}^{1}
$$

In addition, if for any $\delta>0$ we let $\delta \Omega:=\left\{x \in \mathbb{R}^{n}: \frac{x}{\delta} \in \Omega\right\}$ denote $a \delta$ contraction of $\Omega$, then

$$
\mathcal{C}_{\delta \Omega}=\delta^{n} \mathcal{C}_{\Omega}
$$


Proof of Lemma 2.3. A standard application of the direct method goes to show that a minimizer $v$ of $(2.11)$ exists and one readily checks that such a solution satisfies the conditions

$$
\begin{aligned}
& \Delta v=0 \quad \text { in } \Omega, \quad f_{D_{k}} v=a_{k}, \quad \nabla v \cdot \nu=0 \quad \text { on } \partial \Omega \backslash \cup_{k=1}^{N} D_{k}, \\
& \text { and } \nabla v \cdot \nu=\beta_{k} \text { on } D_{k} \quad \text { for some } \beta_{k} \in \mathbb{R}^{1}, k=1, \ldots, N,
\end{aligned}
$$

where $\nu$ denotes a unit normal to $\partial \Omega$. Furthermore, any function $v$ satisfying (2.14) must necessarily be the unique minimizer of (2.11).

Letting $u_{i}$ to be the minimizer of (2.11) subjected to $a_{i}=1, a_{k}=0$ $\forall k \neq i$ we obtain from the above reasoning and the linearity of the problem that $u=\sum_{i=1}^{N} a_{i} u_{i}$ is the minimizer of (2.11) for general constants $a_{1}, \ldots, a_{N}$, so that

$$
C\left(a_{1}, \ldots a_{N}\right)=\sum_{i} \sum_{k} a_{i} a_{k} \int_{\Omega} \nabla u_{i} \cdot \nabla u_{j} d x
$$

and the first part of the lemma follows. The second part follows from applying the transformation

$$
u(x) \rightarrow w(x)=u(x / \delta)
$$

which preserves $f_{D_{k}} u=f_{\delta D_{k}} w$ and transforms a minimizer $u$ of $C_{\Omega}(\mathbf{a})$ to a minimizer $w$ of $C_{\delta \Omega}(\mathbf{a})$, while

$$
\int_{\delta \Omega}|\nabla w|^{2}=\delta^{n-2} \int_{\Omega}|\nabla u|^{2} .
$$

Condition (2.12) is obvious since only a constant minimizer to (2.11) can make $C_{\Omega}(\mathbf{a})$ vanish.

Proof of Proposition 2.1. Let $\tilde{B} \subset \mathbb{R}^{3}$ denote the union of the ball $B(0, b)$ and the $N$ right circular cylinders of radius one, height one, central axis directed along $\Gamma_{k}$ and base passing through the origin. Recalling the definition (2.5) of the set $\tilde{B}_{\varepsilon}$, we observe that $\tilde{B}_{\varepsilon}=\varepsilon^{1+p} \tilde{B}$. Now from (2.6) we see that

$$
\int_{\tilde{B}_{\varepsilon}}\left|\nabla u^{\varepsilon}\right|^{2} \leq \int_{\Omega_{\varepsilon}}\left|\nabla u^{\varepsilon}\right|^{2} \leq C \varepsilon^{2}
$$

If we let

$$
a_{k}^{(\varepsilon)}:=f_{D_{k}^{\varepsilon}} u^{\varepsilon},
$$

then by definition (2.11) we have

$$
\mathbf{a}^{(\varepsilon)} \mathcal{C}_{\varepsilon^{1+p} \tilde{B}} \mathbf{a}^{(\varepsilon), t} \leq \int_{\tilde{B}_{\varepsilon}}\left|\nabla u^{\varepsilon}\right|^{2} \leq C \varepsilon^{2} .
$$


Thus, from (2.13) we obtain

$$
\mathbf{a}^{(\varepsilon)} \mathcal{C}_{\tilde{B}} \mathbf{a}^{(\varepsilon), t} \leq C \varepsilon^{1-p} \rightarrow 0 \quad \text { as } \varepsilon \rightarrow 0
$$

Consequently, after passing to a subsequence if necessary, it follows from (2.12) that for some $\alpha_{0} \in \mathbb{R}^{1}$ one has

$$
\lim _{\varepsilon_{j} \rightarrow 0} \mathbf{a}^{\left(\varepsilon_{j}\right)} \rightarrow \alpha_{0}(1, \ldots, 1)
$$

and the proposition is proved.

We turn next to the compactness of the minimizers $\left\{u^{\varepsilon}\right\}$. More precisely, we wish to identify a subsequential limit that is a function of one variable defined on the graph $\Gamma$. We will accomplish this by considering integral averages of $U_{k}^{\varepsilon}$ (cf. (2.7)) taken over cross-sections of each branch $\Gamma_{k}$. That is, on the $k^{\text {th }}$ branch of $\Omega_{\varepsilon}$ we let $\bar{U}_{k}^{\varepsilon}:\left[\varepsilon^{1+p}, L_{k}\right] \rightarrow \mathbb{R}^{1}$ be defined by

$$
\bar{U}_{k}^{\varepsilon}(s):=f_{\left\{(s, y, z): y^{2}+z^{2}<1\right\}} U_{k}^{\varepsilon}(s, y, z) d y d z .
$$

In the analysis to follow, we will be considering the sequence of measures $\left\{\bar{U}_{k}^{\varepsilon}(s) d s\right\}$ and we wish to emphasize here one of the subtleties of the problem. Because the sequence $\left\{u^{\varepsilon}\right\}$ tends to have a huge gradient near the constriction in the domain $\Omega_{\varepsilon}$, the limit of these measures will have a singular part at the origin. Capturing this singular part is the key to correctly characterizing the limit of minimizers to the original problem. For this reason, we find it convenient to view $\bar{U}_{k}^{\varepsilon}$ as a function defined on $\left[-1, L_{k}\right]$ by extending it to be constant for values below $s=\varepsilon^{1+p}$. Thus, we take

$$
\bar{U}_{k}^{\varepsilon}(s):=\bar{U}_{k}^{\varepsilon}\left(\varepsilon^{1+p}\right) \quad \text { for } \quad-1 \leq s<\varepsilon^{1+p} .
$$

This will ultimately place the support of the singular part of the limiting measure in the interior of the interval of definition rather than having it "leak" out at an endpoint.

2.4 Proposition. There exists a subsequence $\left\{\varepsilon_{j}\right\} \rightarrow 0$ and a function $U^{0} \in$ $H^{1}(\Gamma \backslash\{0\})$ such that for any $\delta>0$ one has

$$
\bar{U}_{k}^{\varepsilon_{j}} \rightarrow U_{k}^{0} \quad \text { on } \quad \delta \leq s \leq L_{k}
$$

weakly in $H^{1}$ and uniformly as $\varepsilon_{j} \rightarrow 0$, where $U_{k}^{0}$ refers to the restriction of $U^{0}$ to $\Gamma_{k}$. 
Furthermore, we have the convergences

$$
\bar{U}_{k}^{\varepsilon_{j}} \rightarrow \begin{cases}U_{k}^{0} & \text { for } s>0 \\ \alpha_{0} & \text { for } s<0\end{cases}
$$

in $L^{1}\left(\left(-1, L_{k}\right)\right)$ and

$$
\left(\bar{U}_{k}^{\varepsilon_{j}}\right)^{\prime} \stackrel{*}{\rightarrow}\left(U_{k}^{0}\right)^{\prime}\left\llcorner\left\{0<s<L_{k}\right\}+\left(U_{k}^{0}(0)-\alpha_{0}\right) \delta_{\{s=0\}},\right.
$$

weakly as measures for each $k \in\{1, \ldots, N\}$ where

$$
U_{k}^{0}(0):=\lim _{s \rightarrow 0^{+}} U_{k}^{0}(s)
$$

Proof. This result follows from ideas developed in [3], [4] and [15], along with the result from Proposition 2.1.

A consequence of (2.8) and the bound (2.6) is the estimate

$$
\begin{aligned}
& \int_{\varepsilon^{1+p}}^{L_{k}} \int_{\left\{y^{2}+z^{2}<1\right\}} a^{\varepsilon}(s)\left|\left(U_{k}^{\varepsilon}\right)_{s}-\frac{\left(g^{\varepsilon}\right)^{\prime}}{g^{\varepsilon}}\left(y\left(U_{k}^{\varepsilon}\right)_{y}+z\left(U_{k}^{\varepsilon}\right)_{z}\right)\right|^{2} d y d z d s \leq \\
& \frac{1}{\varepsilon^{2}} \int_{T_{k}^{\varepsilon}\left(\left\{(s, y, z): \varepsilon^{1+p}<s<L_{k}, y^{2}+z^{2}<1\right\}\right)}\left|\nabla u^{\varepsilon}\right|^{2} d x<C
\end{aligned}
$$

where we have introduced the quantity

$$
a^{\varepsilon}(s):=\frac{g_{\varepsilon}^{2}(s)}{\varepsilon^{2}}
$$

which will play a major role in the analysis to follow.

In view of (2.1) and (2.9), we then note that

$$
\begin{aligned}
& \int_{\varepsilon^{1+p}}^{L_{k}} \int_{\left\{y^{2}+z^{2}<1\right\}} a^{\varepsilon}(s)\left|\frac{\left(g^{\varepsilon}\right)^{\prime}}{g^{\varepsilon}}\left(y\left(U_{k}^{\varepsilon}\right)_{y}+z\left(U_{k}^{\varepsilon}\right)_{z}\right)\right|^{2} d y d z d s \leq \\
& \int_{\varepsilon^{1+p}}^{L_{k}} \int_{\left\{y^{2}+z^{2}<1\right\}}\left(\frac{\left(g^{\varepsilon}\right)^{\prime}}{\varepsilon}\right)^{2}\left|y\left(U_{k}^{\varepsilon}\right)_{y}+z\left(U_{k}^{\varepsilon}\right)_{z}\right|^{2} d y d z d s \leq C \varepsilon^{2-2 p} .
\end{aligned}
$$

Hence, we obtain

$$
\int_{\varepsilon^{1+p}}^{L_{k}} \int_{\left\{y^{2}+z^{2}<1\right\}} a^{\varepsilon}(s)\left|\left(U_{k}^{\varepsilon}\right)_{s}\right|^{2} d y d z d s \leq C
$$

Then it follows from the Cauchy-Schwartz inequality that

$$
\int_{\varepsilon^{1+p}}^{L_{k}} a^{\varepsilon}(s)\left[\left(\bar{U}_{k}^{\varepsilon}\right)^{\prime}\right]^{2} d s \leq \frac{1}{\pi} \int_{\varepsilon^{1+p}}^{L_{k}} \int_{\left\{y^{2}+z^{2}<1\right\}} a^{\varepsilon}(s)\left|\left(U_{k}^{\varepsilon}\right)_{s}\right|^{2} d y d z d s<C .
$$


Fixing any $\delta>0$ we observe that $a^{\varepsilon}(s) \equiv 1$ on $\delta \leq s \leq L_{k}$ for $\varepsilon$ small. Then since $\bar{U}_{k}^{\varepsilon}$ is uniformly bounded by (2.4), we see from (2.24) that the sequence $\left\{\bar{U}_{k}^{\varepsilon}\right\}$ is uniformly bounded in $H^{1}\left(\left(\delta, L_{k}\right)\right)$ with a bound independent of $\delta$. Thus, we obtain an $H^{1}$-subsequential limit $U_{i}^{0}$ as asserted in (2.17), and this limit will be continuous in light of the embedding of continuous (in fact, Hölder continuous) functions in $H^{1}$ in one-dimension, cf. [7] or [17].

To establish more delicate compactness near the constriction, we pause to list the crucial properties of the factor $a^{\varepsilon}$, all of which are easily derivable from the definition of $g_{\varepsilon}$ (cf. (2.1)):

$$
\begin{aligned}
& \int_{\varepsilon^{p}}^{L_{k}} \frac{1}{a^{\varepsilon}(s)} d s \rightarrow L_{k}, \\
& \int_{\varepsilon^{1+p}}^{\varepsilon^{p}} \frac{1}{a^{\varepsilon}(s)} d s \rightarrow \frac{1}{b}, \quad \text { and } \\
& \int_{0}^{\varepsilon^{1+p}} \frac{1}{a^{\varepsilon}(s)} d s \rightarrow 0 \quad \text { as } \quad \varepsilon \rightarrow 0 .
\end{aligned}
$$

In particular, we see that

$$
\int_{\varepsilon^{1+p}}^{L_{k}} \frac{1}{a^{\varepsilon}(s)} d s<C .
$$

Now, writing

$$
\begin{aligned}
& \int_{\varepsilon^{1+p}}^{L_{k}}\left|\left(\bar{U}_{k}^{\varepsilon}\right)^{\prime}\right| d s=\int_{\varepsilon^{1+p}}^{L_{k}} \frac{1}{\sqrt{a^{\varepsilon}(s)}} \sqrt{a^{\varepsilon}(s)}\left|\left(\bar{U}_{k}^{\varepsilon}\right)^{\prime}\right| d s \\
& \leq\left(\int_{\varepsilon^{1+p}}^{L_{k}} \frac{1}{a^{\varepsilon}(s)} d s\right)^{1 / 2}\left(\int_{\varepsilon^{1+p}}^{L_{k}} a^{\varepsilon}(s)\left[\left(\bar{U}_{k}^{\varepsilon}\right)^{\prime}\right]^{2} d s\right)^{1 / 2},
\end{aligned}
$$

we conclude via (2.24) and (2.28) that $\left\{\bar{U}_{k}^{\varepsilon}\right\}$ is uniformly bounded in $B V\left(\left(-1, L_{k}\right)\right)$, in fact in $W^{1,1}\left(\left(-1, L_{k}\right)\right)$, as well. Thus, the sequence has a subsequential limit in $L^{1}\left(\left(-1, L_{k}\right)\right)$, and it must be equal to $\alpha_{0}$ for $s<0$ in view of $(2.16)$ and Lemma 2.1. This yields (2.18). The uniform bound on the $L^{1}$-norms of the sequence $\left\{\left(\bar{U}_{k}^{\varepsilon}\right)^{\prime}\right\}$ implies the weak convergence as measures of a subsequence of derivatives which is the content of assertion (2.19).

We are now able to state our main result characterizing the asymptotic behavior of the minimizers $u^{\varepsilon}$ through a $\Gamma$-convergence type of result saying that the limit minimizes a limiting problem on the graph $\Gamma$.

2.5 Theorem. Let $\left\{u^{\varepsilon}\right\} \in \mathcal{A}_{\varepsilon}$ be any sequence of minimizers of the Dirichlet integral taken over $\Omega_{\varepsilon}$. Then the subsequential limit $U^{0} \in H^{1}(\Gamma \backslash\{0\})$ 
guaranteed by Proposition 2.4 and the value $\alpha_{0}$ emerging in Proposition 2.1 solve the problem

$$
\inf _{U \in \mathcal{A}_{\Gamma}, \alpha \in \mathbb{R}^{1}}\left\{\sum_{k=1}^{N} \int_{\Gamma_{k}}\left(U_{k}^{\prime}\right)^{2} d s+b \sum_{k=1}^{N}\left|U_{k}(0)-\alpha\right|^{2}\right\},
$$

where the admissible set $\mathcal{A}_{\Gamma}$ is given by

$$
\mathcal{A}_{\Gamma}:=\left\{U \in H^{1}(\Gamma \backslash\{0\}): U_{k}\left(L_{k}\right)=c_{k} \text { for } k=1, \ldots, N\right\}
$$

and we have denoted $U_{k}:=U \circ \gamma_{k}$.

2.6 Remark. We observe through minimization over $\alpha$ that the minimizing pair $\left(U^{0}, \alpha_{0}\right)$ are related via the condition

$$
\alpha_{0}=\frac{1}{N} \sum_{k=1}^{N} U_{k}^{0}(0)
$$

2.7 Remark. With a little extra effort, one could establish a full $\Gamma$-convergence result as was done in e.g. [4]. This would involve strengthening (2.30) below to the assertion that

$$
\liminf _{\varepsilon \rightarrow 0} \frac{1}{\varepsilon^{2}} \int_{\Omega_{\varepsilon}}\left|\nabla v^{\varepsilon}\right|^{2} d x \geq \pi\left[\sum_{k=1}^{N} \int_{\Gamma_{k}}\left(V_{k}\right)^{\prime 2} d s+b \sum_{k=1}^{N}\left|V_{k}(0)-\alpha\right|^{2}\right],
$$

for any $V \in H^{1}(\Gamma \backslash\{0\})$, constant $\alpha$ and sequence $\left\{v^{\varepsilon}\right\}$ such that the analogues of the convergences (2.18) and (2.19) are satisfied. One motivation for pursuing this would be to look for possible local minimizers of the original problem (2.3).

2.8 Remark. There are numerous fairly obvious generalizations of Theorem 2.5 that one can similarly obtain. Certainly such a result will hold in $n$ dimensions as well. One could also take non-constant Dirichlet data $c_{k}(x)$ with the limiting energy picking up the average for its data. Another allowable generalizations would involve replacing the Laplacian by a more general elliptic operator. Also, one could take the constrictions to differ from each other in geometry. This could most easily be accomplished by replacing $b$ in definition (2.1) by $b_{i}$ along each branch. Then the limiting energy would take the form

$$
\sum_{k=1}^{N} \int_{\Gamma_{k}}\left(U_{k}^{\prime}\right)^{2} d s+\sum_{k=1}^{N} b_{k}\left|U_{k}(0)-\alpha\right|^{2}
$$


Finally, without any substantive change, one could phrase the result on more general graphs, that is, structures consisting of finitely many smooth curves joined at finitely many nodes. In the next section, we will discuss a generalization to the setting of the Ginzburg-Landau energy along these lines.

2.9 Remark. The severity of the constriction is controlled by the parameter $p$ appearing in the definition of $g^{\varepsilon}$, which we take to satisfy $0<p<1$. If one instead considers a more extreme constriction by taking $p>1$ then it is not hard to check that the limiting variational problem will include no jump term at the junction whatsoever. In other words, a limit of minimizers $U^{0}$ would then simply minimize the functional

$$
\sum_{k=1}^{N} \int_{\Gamma_{k}}\left(U_{k}^{\prime}\right)^{2} d s
$$

and there would be no transmission across the origin of the graph. Consequently, $U^{0}$ would equal the constant $c_{k}$ on $\Gamma_{k}$ for each $k$. We did not explore the critical case $p=1$.

Proof. The identification of $U^{0}$ as a minimizer of (2.29) will result from establishing the following two claims. First we will show that

$$
\liminf _{\varepsilon_{j} \rightarrow 0} \frac{1}{\varepsilon_{j}^{2}} \int_{\Omega_{\varepsilon_{j}}}\left|\nabla u^{\varepsilon_{j}}\right|^{2} d x \geq \pi\left[\sum_{k=1}^{N} \int_{\Gamma_{k}}\left(U_{k}^{0}\right)^{\prime 2} d s+b \sum_{k=1}^{N}\left|U_{k}^{0}(0)-\alpha_{0}\right|^{2}\right],
$$

where $\alpha_{0}$ is the value emerging in Lemma 2.1. Then we will show that for any $V \in \mathcal{A}_{\Gamma}$, and for any $\alpha \in \mathbb{R}^{1}$, there exists a sequence $\left\{v^{\varepsilon}\right\} \subset H^{1}\left(\Omega_{\varepsilon}\right)$ such that

$$
\lim _{\varepsilon \rightarrow 0} \frac{1}{\varepsilon^{2}} \int_{\Omega_{\varepsilon}}\left|\nabla v^{\varepsilon}\right|^{2} d x=\pi\left[\sum_{k=1}^{N} \int_{\Gamma_{k}} V_{k}^{\prime 2} d s+b \sum_{k=1}^{N}\left|V_{k}(0)-\alpha\right|^{2}\right],
$$

where we denote by $V_{k}$ the restriction of $V$ to the branch $\Gamma_{k}$. Using the minimizing property of the sequence $\left\{u^{\varepsilon}\right\}$, we can then combine (2.30) and (2.31) to conclude that $U^{0}$ solves (2.29) as asserted, namely

$$
\sum_{k=1}^{N} \int_{\Gamma_{k}}\left(U_{k}^{0}\right)^{\prime 2} d s+b \sum_{k=1}^{N}\left|U_{k}^{0}(0)-\alpha_{0}\right|^{2} \leq \sum_{k=1}^{N} \int_{\Gamma_{k}} V_{k}^{\prime 2} d s+b \sum_{k=1}^{N}\left|V_{k}(0)-\alpha\right|^{2},
$$

for arbitrary $V \in H^{1}(\Gamma \backslash\{0\})$ and $\alpha \in \mathbb{R}^{1}$. 
Proof of Claim (2.30): To establish (2.30), we first invoke (2.21), (2.22) and (2.24) to obtain

$$
\frac{1}{\varepsilon^{2}} \int_{\Omega_{\varepsilon}}\left|\nabla u^{\varepsilon}\right|^{2} d x \geq \pi \sum_{k=1}^{N} \int_{\varepsilon^{1+p}}^{L_{k}} a_{\varepsilon}(s)\left[\left(\bar{U}_{k}^{\varepsilon}\right)^{\prime}\right]^{2} d s .
$$

Let us now denote by $\mu_{k}^{\varepsilon}$ and $\nu_{\bar{U}_{k}^{\varepsilon}}$ the measures on $\left[-1, L_{k}\right]$ given by

$$
d \mu_{k}^{\varepsilon}(s):=\left\{\begin{array}{cl}
\frac{1}{a^{\varepsilon}(s)} d s & \text { for } s \geq 0 \\
1 d s & \text { for } s<0
\end{array} \quad \text { and } \quad d \nu_{\bar{U}_{k}^{\varepsilon}}(s):=\left(\bar{U}_{k}^{\varepsilon}\right)^{\prime}(s) d s\right.
$$

respectively. Using the constancy of $\bar{U}_{k}^{\varepsilon}$ for $s<\varepsilon^{1+p}$, this allows us to write

$$
\int_{\varepsilon^{1+p}}^{L_{k}} a^{\varepsilon}(s)\left|\left(\bar{U}_{k}^{\varepsilon}\right)^{\prime}\right|^{2} d s=\int_{-1}^{L_{k}}\left|\frac{d \nu_{\bar{U}_{k}^{\varepsilon}}}{d \mu_{k}^{\varepsilon}}\right|^{2} d \mu_{k}^{\varepsilon}(s) \quad \text { for each } k,
$$

where in the last integral the quantity $\frac{d \nu_{\bar{U}}^{\varepsilon}}{d \mu_{k}^{\varepsilon}}$ refers to the (Radon-Nikodym) derivative of $\nu_{\bar{U}_{k}^{\varepsilon}}$ with respect to $\mu_{k}^{\varepsilon}$.

Then, using the definition of $g^{\varepsilon}$ and $a^{\varepsilon}$ one can easily check that

$$
d \mu_{k}^{\varepsilon}(s) \stackrel{*}{\rightarrow} 1 d s+\frac{1}{b} \delta_{\{s=0\}}=: d \mu_{k}^{0}
$$

Also, recall that (2.19) asserts the convergence

$$
d \nu_{\bar{U}_{k}^{\varepsilon_{j}}} \stackrel{*}{\rightarrow}\left(U_{k}^{0}\right)^{\prime}(s) d s\left\llcorner\{s>0\}+\left(U_{k}^{0}(0)-\alpha_{0}\right) \delta_{\{s=0\}}=: d \nu_{U_{k}^{0}} .\right.
$$

Hence, invoking Theorem 3.1 of [3], we conclude that

$$
\begin{aligned}
& \liminf _{\varepsilon_{j} \rightarrow 0} \sum_{i=1}^{N} \int_{-1}^{L_{k}}\left|\frac{d \nu_{\bar{U}_{k}}^{\varepsilon_{j}}}{d \mu_{\varepsilon_{j}}}\right|^{2} d \mu_{k}^{\varepsilon_{j}}(s) \geq \sum_{k=1}^{N} \int_{-1}^{L_{k}}\left|\frac{d \nu_{U_{k}^{0}}}{d \mu_{k}^{0}}\right|^{2} d \mu_{k}^{0}(s) \\
& =\sum_{k=1}^{N} \int_{\Gamma_{i}}\left|\left(U_{k}^{0}\right)^{\prime}\right|^{2} d s+b\left|U_{k}^{0}(0)-\alpha_{0}\right|^{2} .
\end{aligned}
$$

Combining this last inequality with (2.32) and (2.33), we obtain (2.30).

Proof of Claim (2.31): Fix any function $V \in \mathcal{A}_{\Gamma}$ and any $\alpha \in \mathbb{R}^{1}$. The construction is similar to that used in [15]. We introduce the function $\lambda^{\varepsilon}$ : $[0, \infty) \rightarrow \mathbb{R}^{1}$ via the formula

$$
\frac{1}{a^{\varepsilon}(s)}=1+\lambda^{\varepsilon}(s) \text {. }
$$

We observe from (2.34) that $\lambda^{\varepsilon}$ behaves like a $\delta$-function at the origin with mass $1 / b$. In particular, if we introduce

$$
\beta_{k}^{\varepsilon}:=\int_{\varepsilon^{1+p}}^{L_{k}} \lambda^{\varepsilon}(s) d s
$$


then from (2.25)-(2.26) we see that $\beta_{k}^{\varepsilon} \rightarrow \frac{1}{b}$ as $\varepsilon \rightarrow 0$.

Now we can define a sequence $v^{\varepsilon} \in H^{1}\left(\Omega_{\varepsilon}\right)$ that will serve to verify (2.31). Recalling the definition of $\tilde{B}_{\varepsilon}$ given below (2.4), we set $v^{\varepsilon} \equiv \alpha$ in $\tilde{B}_{\varepsilon}$. Then, on the $k^{\text {th }}$ branch of $\Omega_{\varepsilon}$ outside of $\tilde{B}_{\varepsilon}$, we define $v^{\varepsilon}=V_{k}^{\varepsilon}(s)$ (that is, independent of $y$ and $z$ ) by

$$
V_{k}^{\varepsilon}(s)=V_{k}(s)+\frac{1}{\beta_{k}^{\varepsilon}}\left(\alpha-V_{k}\left(\varepsilon^{1+p}\right)\right) \int_{s}^{L_{k}} \lambda^{\varepsilon}\left(s^{\prime}\right) d s^{\prime} \quad \text { for } \quad \varepsilon^{1+p} \leq s \leq L_{k} .
$$

Observe, in light of (2.35), that $V_{k}^{\varepsilon}(s)=V_{k}(s)$ for $s>\varepsilon^{p}$. We now essentially follow the calculation from [15]. We present it here to keep the treatment self-contained. Through the change of variables formula (2.8) employed for a function of $s$ only, and through extensive use of (2.35), one finds

$$
\begin{aligned}
& \frac{1}{\pi} \lim _{\varepsilon \rightarrow 0} \frac{1}{\varepsilon^{2}} \int_{\Omega_{\varepsilon}}\left|\nabla v^{\varepsilon}\right|^{2} d x=\frac{1}{\pi} \lim _{\varepsilon \rightarrow 0} \frac{1}{\varepsilon^{2}} \int_{\Omega_{\varepsilon} \backslash \tilde{B}_{\varepsilon}}\left|\nabla v^{\varepsilon}\right|^{2} d x= \\
& \lim _{\varepsilon \rightarrow 0} \sum_{k=1}^{N} \int_{\varepsilon^{1+p}}^{L_{k}} \frac{g_{\varepsilon}^{2}(s)}{\varepsilon^{2}}\left|\left(V_{k}^{\varepsilon}\right)_{s}\right|^{2} d s= \\
& \lim _{\varepsilon \rightarrow 0} \sum_{k=1}^{N} \int_{\varepsilon^{1+p}}^{L_{k}} a_{\varepsilon}(s)\left|V_{k}^{\prime}(s)-\frac{1}{\beta_{k}^{\varepsilon}}\left(\alpha-V_{k}(0)\right) \lambda^{\varepsilon}(s)\right|^{2} d s= \\
& \lim _{\varepsilon \rightarrow 0} \sum_{k=1}^{N}\left[\int_{\varepsilon^{1+p}}^{L_{k}} a_{\varepsilon}(s)\left|V_{k}^{\prime}\right|^{2} d s+\frac{1}{\left(\beta_{k}^{\varepsilon}\right)^{2}}\left(V_{k}(0)-\alpha\right)^{2} \int_{\varepsilon^{1+p}}^{L_{k}} a_{\varepsilon}(s) \lambda^{\varepsilon}(s)^{2} d s\right. \\
& \left.+\frac{2\left(V_{k}(0)-\alpha\right)}{\beta_{k}^{\varepsilon}} \int_{\varepsilon^{1+p}}^{L_{k}} V_{k}^{\prime}(s) a_{\varepsilon}(s) \lambda^{\varepsilon}(s) d s\right]=\lim _{\varepsilon \rightarrow 0} \sum_{k=1}^{N} I+I I+I I I .
\end{aligned}
$$

Since $a^{\varepsilon} \rightarrow 1$ in $L^{2}\left(\left(0, L_{k}\right)\right)$, one immediately sees that

$$
\lim _{\varepsilon \rightarrow 0} I=\int_{0}^{L_{k}}\left|V_{k}^{\prime}\right|^{2} d s
$$

Then, since

$$
\int_{\varepsilon^{1+p}}^{L_{k}} a_{\varepsilon}(s) \lambda^{\varepsilon}(s)^{2} d s=\int_{\varepsilon^{1+p}}^{L_{k}}\left(1-a_{\varepsilon}(s)\right) \lambda^{\varepsilon}(s) d s=\beta_{k}^{\varepsilon}-\int_{\varepsilon^{1+p}}^{L_{k}}\left(1-a_{\varepsilon}(s)\right) d s
$$

we can use the fact that $\beta_{k}^{\varepsilon} \rightarrow \frac{1}{b}$ to check that

$$
\lim _{\varepsilon \rightarrow 0} I I=b\left(V_{k}(0)-\alpha\right)^{2} .
$$

Finally, the estimate

$$
\begin{aligned}
& \left|\frac{2\left(V_{k}(0)-\alpha\right)}{\beta_{k}^{\varepsilon}} \int_{\varepsilon^{1+p}}^{L_{k}} V_{k}^{\prime}(s) a_{\varepsilon}(s) \lambda^{\varepsilon}(s) d s\right| \leq C \int_{\varepsilon^{1+p}}^{L_{k}}\left|V_{k}^{\prime}(s)\left(1-a_{\varepsilon}(s)\right)\right| d s \\
& \leq C\left(\int_{\varepsilon^{1+p}}^{L_{k}}\left|V_{k}^{\prime}\right|^{2} d s\right)^{1 / 2}\left(\int_{\varepsilon^{1+p}}^{L_{k}}\left(1-a_{\varepsilon}(s)\right)^{2} d s\right)^{1 / 2} \rightarrow 0,
\end{aligned}
$$


implies that $\lim _{\varepsilon \rightarrow 0} I I I=0$ and we return to (2.37) to obtain (2.31).

We conclude this section with a discussion of the minimization problem (2.29) solved by $U^{0}$ and $\alpha=\alpha_{0}=\frac{1}{N} \sum_{k=1}^{N} U_{k}^{0}(0)$. Taking a first variation in the variable $U$ on each branch, that is substituting in $U_{k}^{0}+\delta W_{k}$ on $\Gamma_{k}$ for $W_{k} \in H^{1}\left(\Gamma_{k}\right)$ with $W_{k}\left(L_{k}\right)=0$ and setting the $\delta$-derivative of the energy equal to zero, one obtains

$$
\sum_{k=1}^{N} \int_{0}^{L_{k}}\left(U_{k}^{0}\right)^{\prime} W_{k}^{\prime} d s+b \sum_{k=1}^{N}\left(U_{k}^{0}(0)-\alpha_{0}\right) W_{k}(0)=0 .
$$

Integrating by parts, we conclude, not surprisingly, that $U^{0}$ is linear on each branch. Then, taking $W_{k}(0)=0$ for all $k$ except $k=j$ for some $j \in$ $\{1, \ldots, N\}$ we obtain $N$ natural boundary conditions at the origin of the graph:

$$
\left(U_{j}^{0}\right)^{\prime}(0)=b\left(U_{j}^{0}(0)-\alpha_{0}\right) \quad \text { for } \quad j=1, \ldots, N .
$$

Recalling that $\alpha_{0}$ is simply the average of the values $\left\{U_{k}^{0}(0)\right\}$, we note that summing over the $k$ conditions in (2.39) leads to a zero net flux condition at the origin of the graph

$$
\sum_{k=1}^{N}\left(U_{k}^{0}\right)^{\prime}(0)=0
$$

Since the $U_{k}^{0}$ are linear, one can view (2.39) as a system of $N$ linear equations for the $N$ unknown slopes, namely

$$
\frac{c_{k}-U_{k}(0)}{L_{k}}=b\left(U_{k}(0)-\frac{1}{N} \sum_{k^{\prime}=1}^{N} U_{k^{\prime}}(0)\right) .
$$

Finally, we note that even in the most trivial case $N=2$, the solution will not in general be continuous at the origin. For example, taking $b=L_{1}=$ $L_{2}=1$ one can explicitly calculate the minimizer $U^{0}$ to be given by

$$
U_{1}^{0}\left(s_{1}\right)=\frac{1}{4}\left(c_{1}-c_{2}\right)\left(s_{1}-1\right)+c_{1}, \quad U_{2}^{0}\left(s_{2}\right)=-\frac{1}{4}\left(c_{1}-c_{2}\right)\left(s_{2}-1\right)+c_{2}
$$

so that $U_{1}^{0}(0)-U_{2}^{0}(0)=\frac{1}{2}\left(c_{1}-c_{2}\right)$.

\section{Generalization to Ginzburg-Landau along a network of constricted arcs.}

In this section we pursue a generalization of the results of the previous section in various directions by extending this analysis to the case of the Ginzburg- 
Landau energy as was accomplished in [15]. In [15], we took the limiting graph forming the skeleton of the domain $\Omega_{\varepsilon}$ to be one circle and the domain possessed one constriction (one node on the circle). Also, in [15] we took an applied magnetic field directed depending only on $x_{1}$ and $x_{2}$ and directed along the $x_{3}$-axis. Here we wish to consider domains that shrink in the $\varepsilon \rightarrow 0$ limit to graphs consisting of a finite number of smooth $\operatorname{arcs}$ in $\mathbb{R}^{3}$ joined at a finite number of nodes, and we will consider arbitrary smooth applied magnetic fields. Let us first introduce the Ginzburg-Landau model for superconductivity.

We will use the following non-dimensional version of the Ginzburg-Landau energy functional taken over a sample $\Omega_{\varepsilon} \subset \mathbb{R}^{3}$ to be described shortly:

$$
\begin{array}{r}
G_{\varepsilon}(\psi, \mathbf{A})=\frac{1}{\varepsilon^{2}} \int_{\Omega_{\varepsilon}}\left(|(\nabla-i \mathbf{A}) \psi|^{2}+\frac{\tilde{\nu}^{2}}{2}\left(|\psi|^{2}-\mu^{2}\right)^{2}\right) d x+ \\
\frac{1}{\varepsilon^{2}} \int_{\mathbb{R}^{3}}\left|\nabla \times \mathbf{A}-\mathbf{H}^{e}\right|^{2} d x .
\end{array}
$$

Here $\Psi: \Omega_{\varepsilon} \rightarrow \mathbb{C}$ is the order parameter whose square modulus measures the superconducting electron density, $\mathbf{A}: \mathbb{R}^{3} \rightarrow \mathbb{R}^{3}$ is the effective magnetic potential associated with the effective magnetic field $\mathbf{H}$ through $\nabla \times \mathbf{A}=\mathbf{H}$, and $\mathbf{H}^{e}: \mathbb{R}^{3} \rightarrow \mathbb{R}^{3}$ is an arbitrary given, smoothly varying, applied magnetic field. The quantities $\tilde{\nu}$ and $\mu$ are material parameters with $\mu^{2}$ proportional to the difference between the critical temperature $T_{c}$ and the temperature of the sample. We assume we are in the superconducting temperature regime where this difference is positive. We have retained $\mu$ in the formulation in order to highlight the possibility of using it as a bifurcation parameter in future studies of the onset problem. Note that the energy $G_{\varepsilon}$ has already been scaled so that the minimum energy remains uniformly bounded away from both zero and infinity for small $\varepsilon$.

Next we describe the sample $\Omega_{\varepsilon}$. We begin with a graph $\Gamma$ consisting of a finite number of $C^{2} \operatorname{arcs} \Gamma_{k}, k=1, \ldots, N$ joined at points $\left\{P_{l}\right\}, l=1, \ldots, M$. For each $k$, we denote by $\gamma_{k}:\left[0, L_{k}\right] \rightarrow \mathbb{R}^{3}$ a smooth parametrization of $\Gamma_{k}$ by arclength, and we allow for the possibility that $\gamma_{k}(0)=\gamma_{k}\left(L_{k}\right)$, in which case, $\gamma_{k}^{\prime}(0)$ is not necessarily assumed to be parallel to $\gamma^{\prime}\left(L_{k}\right)$. See Figure 2 . As in Section 2, the domain $\Omega_{\varepsilon}$ arises through a "thickening" of this graph by taking the union of a tubular neighborhood of $\Gamma$ along with small balls centered at the points $\left\{P_{l}\right\}$. To make this precise we introduce a function $g_{k}^{\varepsilon}(s)$ to govern the thickness of the $k^{\text {th }}$ branch of $\Omega_{\varepsilon}$ as an even extension of 


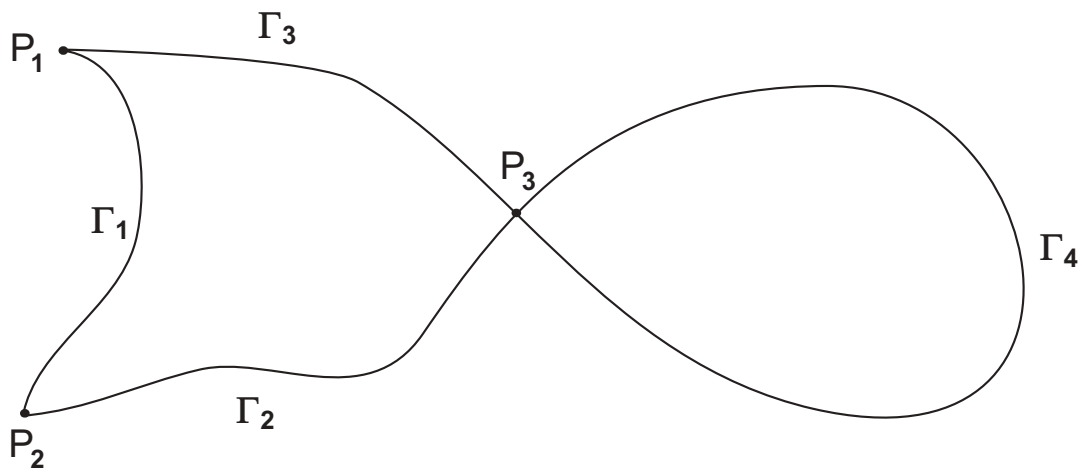

Figure 2: A general graph $\Gamma$ in $\mathbb{R}^{3}$.

the function $g^{\varepsilon}$ defined in (2.1) via the formula

$$
g_{k}^{\varepsilon}(s)=\left\{\begin{array}{cc}
g^{\varepsilon}(s) & \text { for } \quad 0 \leq s \leq \frac{L_{k}}{2} \\
g^{\varepsilon}\left(L_{k}-s\right) & \text { for } \quad \frac{L_{k}}{2} \leq s \leq L_{k} .
\end{array}\right.
$$

Then we define the $k t h$ branch, $C_{k}^{\varepsilon}$ of $\Omega_{\varepsilon}$ via

$$
C_{k}^{\varepsilon}:=\left\{x:\left(x_{1}, x_{2}, x_{3}\right)=T_{\varepsilon}^{k}(s, y, z) \text { for }(s, y, z) \in \mathcal{C}_{k}\right\} \text {, }
$$

where $\mathcal{C}_{k}:=\left\{(s, y, z): 0 \leq s \leq L_{k}, y^{2}+z^{2}<1\right\}$ and where $T_{k}^{\varepsilon}: \mathcal{C}_{k} \rightarrow \mathbb{R}^{3}$ is given by

$$
T_{k}^{\varepsilon}(s, y, z)=\gamma_{k}(s)+y g_{k}^{\varepsilon}(s) \mathbf{n}_{k}(s)+z g_{k}^{\varepsilon}(s) \mathbf{b}_{k}(s) .
$$

Here $\mathbf{n}_{k}$ denotes the normal to the arc $\Gamma_{k}$ and $\mathbf{b}_{k}$ denotes the binormal in a standard Frenet frame. For future reference, we recall the Frenet equations that serve to define the curvature $\kappa_{k}$ and torsion $\tau_{k}$ of the $k^{t h}$ arc $\Gamma_{k}$ :

$\gamma_{k}^{\prime \prime}(s)=\kappa_{k}(s) \mathbf{n}_{k}(s), \quad \mathbf{n}_{k}^{\prime}(s)=-\kappa_{k}(s) \gamma_{k}^{\prime}(s)-\tau_{k}(s) \mathbf{b}_{k}(s), \quad \mathbf{b}_{k}^{\prime}(s)=-\tau_{k}(s) \mathbf{n}_{k}(s)$.

3.1 Remark. In case a particular arc, or section of an arc, consists of a line segment, of course the frame is not well-defined. In this case, however, we simply use a coordinate system such as that used in Section 2. This does not affect the analysis and we will not comment further on this issue.

Now, as in the previous section, we define $\Omega_{\varepsilon}$ as the union of these $N$ branches described above with the $M$ balls $B\left(P_{l}, b \varepsilon^{1+p}\right)$, where for every $l$ we have at least one $k$ such that $P_{l}=\gamma_{k}(0)$ or $P_{l}=\gamma_{k}\left(L_{k}\right)$ or both. (See Figure 3). 


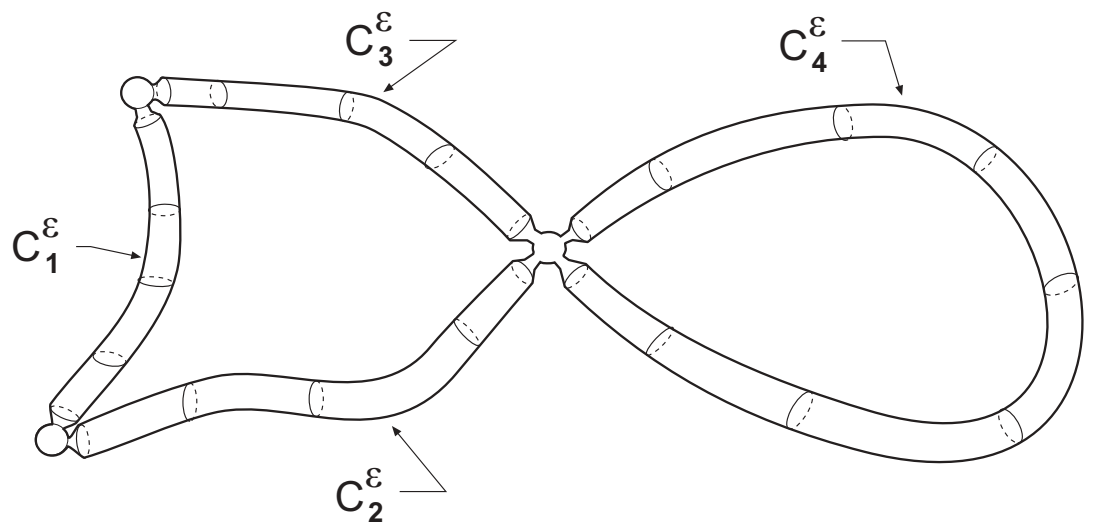

Figure 3: A domain $\Omega_{\varepsilon}$ consisting of multiple branches constricted at a node. The pipes $C_{1}^{\varepsilon}, \ldots, C_{4}^{\varepsilon}$ are tapered tubular neighborhoods of the branches $\Gamma_{k}$ of the graph $\Gamma$.

Our goal in this section is again to characterize the asymptotic behavior of minimizers. To set up the minimization of $G_{\varepsilon}$ in the proper function spaces, we introduce the space $\mathcal{H}$ as the completion of the set

$$
\left\{\phi \in C^{\infty}\left(\mathbb{R}^{3} ; \mathbb{R}^{3}\right): \phi \text { compactly supported }\right\}
$$

with respect to the norm $\|\nabla \phi\|_{L^{2}\left(\mathbb{R}^{3} ; \mathbb{R}^{3}\right)}=\left(\int_{\mathbb{R}^{3}}|\nabla \phi|^{2} d x\right)^{1 / 2}$. Then we define $\mathcal{H}_{0}$ to be

$$
\mathcal{H}_{0}=\{\phi \in \mathcal{H}: \operatorname{div} \phi=0\},
$$

and consider competitors $\mathbf{A}$ satisfying $\mathbf{A}-\mathbf{A}^{e} \in \mathcal{H}_{0}$, where $\mathbf{A}^{e}$ is the applied magnetic potential satisfying

$$
\nabla \times \mathbf{A}^{e}=\mathbf{H}^{e} \quad \text { and } \operatorname{div} \mathbf{A}^{e}=0 \quad \text { in } \mathbb{R}^{3}, .
$$

We summarize below two results we will need about minimizers of the Ginzburg-Landau energy.

3.2 Theorem. For all positive $\varepsilon<1$, there exists a pair $\left(\psi^{\varepsilon}, \mathbf{A}^{\varepsilon}\right)$ solving the variational problem

$$
\inf _{\left\{\psi \in H^{1}\left(\Omega_{\varepsilon} ; \mathbb{C}\right), \mathbf{A}-\mathbf{A}^{e} \in \mathcal{H}_{0}\right\}} G_{\varepsilon}(\psi, \mathbf{A}) .
$$

The function $\psi^{\varepsilon}$ is smooth in $\Omega_{\varepsilon}$ while the function $\mathbf{A}^{\varepsilon}$ is smooth in $\mathbb{R}^{3} \backslash \partial \Omega_{\varepsilon}$ and continuously differentiable across $\partial \Omega_{\varepsilon}$. Furthermore, the mini- 
mizers satisfy the Ginzburg-Landau system

$$
\begin{aligned}
& \left(\nabla-i \mathbf{A}^{\varepsilon}\right)^{2} \psi^{\varepsilon}=\tilde{\nu}^{2}\left(\left|\psi^{\varepsilon}\right|^{2}-\mu^{2}\right) \psi^{\varepsilon} \quad \text { in } \quad \Omega_{\varepsilon}, \\
& \nabla \times \nabla \times\left(\mathbf{A}^{\varepsilon}-\mathbf{A}^{e}\right) \quad\left(=-\Delta\left(\mathbf{A}^{\varepsilon}-\mathbf{A}^{e}\right)\right)= \\
& \left\{\begin{array}{cr}
\frac{i}{2}\left(\left(\psi^{\varepsilon}\right)^{*} \nabla \psi^{\varepsilon}-\psi^{\varepsilon} \nabla\left(\psi^{\varepsilon}\right)^{*}\right)-\left|\psi^{\varepsilon}\right|^{2} \mathbf{A}^{\varepsilon} & \text { for } x \in \Omega_{\varepsilon} \\
0 & \text { for } x \in \mathbb{R}^{3} \backslash \overline{\Omega_{\varepsilon}}
\end{array}\right.
\end{aligned}
$$

and the boundary condition

$$
\left(\nabla-i \mathbf{A}^{\varepsilon}\right) \psi^{\varepsilon} \cdot \nu^{\varepsilon}=0 \quad \text { on } \quad \partial \Omega_{\varepsilon} .
$$

Here $\cdot{ }^{*}$ denotes complex conjugation and $\nu^{\varepsilon}$ denotes the outer unit normal along $\partial \Omega_{\varepsilon}$.

Finally, the order parameter $\psi^{\varepsilon}$ satisfies the condition

$$
\left|\psi^{\varepsilon}\right| \leq \mu \quad \text { in } \quad \bar{\Omega}_{\varepsilon} .
$$

Existence follows from a standard application of the direct method to the Ginzburg-Landau energy; such an apporach can be found for instance in [6] or [14]. The regularity theory follows from standard elliptic theory, which in this context can be found for instance in [8]. Inequality (3.10) is an easy consequence of the maximum principle, see e.g. [6].

3.3 Proposition. There exist positive constants $C_{1}$ and $C_{2}$ independent of E such that

$$
\begin{aligned}
& G_{\varepsilon}\left(\psi^{\varepsilon}, \mathbf{A}^{\varepsilon}\right) \leq C_{1} \quad \text { and } \\
& \int_{\Omega_{\varepsilon}}\left|\nabla \psi^{\varepsilon}\right|^{2} d x \leq C_{2} \varepsilon^{2} .
\end{aligned}
$$

Furthermore, one has the uniform convergence

$$
\left\|\mathbf{A}^{\varepsilon}-\mathbf{A}^{e}\right\|_{L^{\infty}\left(B_{R}(0) ; \mathbb{R}^{3}\right)} \rightarrow 0 \quad \text { as } \quad \varepsilon \rightarrow 0 \quad \text { for every } R>0
$$

where $B_{R}(0)=\left\{x \in \mathbb{R}^{3}:|x|<R\right\}$. Condition (3.13) in particular implies that

$$
\sup _{(s, y, z) \in \mathfrak{C}_{i}}\left|\mathbf{A}^{\varepsilon}\left(T_{k}^{\varepsilon}(s, y, z)\right)-\mathbf{A}^{e}\left(T_{k}^{\varepsilon}(s, 0,0)\right)\right| \rightarrow 0 \quad \text { as } \quad \varepsilon \rightarrow 0 .
$$

The inequality (3.11) follows by comparing the minimal energy to that of the admissible pair $\left(\mu, \mathbf{A}^{e}\right)$. The proof of the rest of the proposition is identical to that of Proposition 2.2 of [15]. 
Before proceeding we will need to convert the term arising in the GinzburgLandau energy involving integration of the expression $\left|\left(\nabla-i \mathbf{A}^{\varepsilon}\right) \psi^{\varepsilon}\right|^{2}$ over the $k^{\text {th }}$ branch of $\Omega_{\varepsilon}$ into an integral phrased in terms of the coordinates $(s, y, z)$ and in terms of the orthonormal frame $\left(\gamma_{k}^{\prime}, \mathbf{n}_{k}, \mathbf{b}_{k}\right)$. To this end, we introduce $\Psi_{k}^{\varepsilon}: \mathcal{C}_{k} \rightarrow \mathbb{C}$ via

$$
\Psi_{k}^{\varepsilon}(s, y, z)=\psi^{\varepsilon}\left(T_{k}^{\varepsilon}(s, y, z)\right)
$$

Then we use (3.3) and (3.4) to calculate

$$
\begin{aligned}
& \left(\Psi_{k}^{\varepsilon}\right)_{s}=\zeta \nabla_{x} \psi^{\varepsilon} \cdot \gamma_{k}^{\prime}+\delta \nabla_{x} \psi^{\varepsilon} \cdot \mathbf{n}_{k}+\theta \nabla \psi^{\varepsilon} \cdot \mathbf{b}_{k} \\
& \left(\Psi^{\varepsilon}\right)_{y}=g_{k}^{\varepsilon} \nabla_{x} \psi^{\varepsilon} \cdot \mathbf{n}_{k} \quad \text { and } \\
& \left(\Psi_{k}^{\varepsilon}\right)_{z}=g_{k}^{\varepsilon} \nabla_{x} \psi^{\varepsilon} \cdot \mathbf{b}_{k},
\end{aligned}
$$

where we define

$$
\begin{aligned}
& \zeta=\zeta(s, y, \varepsilon, k):=1-\kappa_{k}(s) y g_{k}^{\varepsilon}(s), \\
& \delta=\delta(s, y, z, \varepsilon, k):=y\left(g_{k}^{\varepsilon}\right)^{\prime}(s)-z g_{k}^{\varepsilon}(s) \tau_{k}(s), \\
& \theta=\theta(s, y, z, \varepsilon, k):=z\left(g_{k}^{\varepsilon}\right)^{\prime}(s)-y g_{k}^{\varepsilon}(s) \tau_{k}(s) .
\end{aligned}
$$

From here it easily follows that

$$
\nabla_{x} \psi^{\varepsilon}=\left(\frac{1}{\zeta}\left(\Psi_{k}^{\varepsilon}\right)_{s}-\frac{\delta}{\zeta g_{k}^{\varepsilon}}\left(\Psi_{k}^{\varepsilon}\right)_{y}-\frac{\theta}{\zeta g_{k}^{\varepsilon}}\left(\Psi_{k}^{\varepsilon}\right)_{z}\right) \gamma_{k}^{\prime}+\frac{1}{g_{k}^{\varepsilon}}\left(\Psi_{k}^{\varepsilon}\right)_{y} \mathbf{n}_{k}+\frac{1}{g_{k}^{\varepsilon}}\left(\Psi_{k}^{\varepsilon}\right)_{z} \mathbf{b}_{k} .
$$

Using the fact that the Jacobian of the transformation $T_{k}^{\varepsilon}$ is given by

$$
J\left(T_{k}^{\varepsilon}\right)=\left(g_{k}^{\varepsilon}\right)^{2} \zeta
$$

we then conclude from (3.11) that

$$
\begin{aligned}
& C_{1} \geq \frac{1}{\varepsilon^{2}} \int_{T_{k}^{\varepsilon}\left(\mathfrak{C}_{k}\right)}\left|\left(\nabla-i \mathbf{A}^{\varepsilon}\right) \psi^{\varepsilon}\right|^{2} d x= \\
& \int_{\mathfrak{C}_{k}}\left|\left(\frac{1}{\zeta}\left(\Psi_{k}^{\varepsilon}\right)_{s}-i \mathcal{A}^{\varepsilon, T} \Psi_{k}^{\varepsilon}\right)-\left(\frac{\delta}{\zeta g_{k}^{\varepsilon}}\left(\Psi_{k}^{\varepsilon}\right)_{y}+\frac{\theta}{\zeta g_{k}^{\varepsilon}}\left(\Psi_{k}^{\varepsilon}\right)_{z}\right)\right|^{2} \frac{\left(g_{k}^{\varepsilon}\right)^{2} \zeta}{\varepsilon^{2}} d s d y d z \\
& +\int_{\mathfrak{C}_{k}}\left\{\left|\left(\Psi_{k}^{\varepsilon}\right)_{y}-i g_{k}^{\varepsilon} \mathcal{A}^{\varepsilon, n} \Psi_{k}^{\varepsilon}\right|^{2}+\left|\left(\Psi_{k}^{\varepsilon}\right)_{z}-i g_{k}^{\varepsilon} \mathcal{A}^{\varepsilon, b} \Psi_{k}^{\varepsilon}\right|^{2}\right\} \frac{\zeta}{\varepsilon^{2}} d s d y d z,
\end{aligned}
$$

where we have introduced

$$
\mathcal{A}^{\varepsilon, T}:=\mathbf{A}^{\varepsilon} \cdot \gamma_{k}^{\prime}, \quad \mathcal{A}^{\varepsilon, n}:=\mathbf{A}^{\varepsilon} \cdot \mathbf{n}_{k} \quad \text { and } \quad \mathcal{A}^{\varepsilon, b}:=\mathbf{A}^{\varepsilon} \cdot \mathbf{b}_{k}
$$


to denote the tangential, normal and binormal components of the minimizing potential $\mathbf{A}^{\varepsilon}$. Since (3.14) implies a uniform bound on $\mathcal{A}^{\varepsilon, n}$ and $\mathcal{A}^{\varepsilon, b}$, and (3.15) implies e.g. that $\zeta \geq \frac{1}{2}$, one immediate consequence of (3.19) along with (3.10) is that

$$
\int_{\mathbb{C}_{k}}\left(\left(\Psi_{k}^{\varepsilon}\right)_{y}\right)^{2}+\left(\left(\Psi_{k}^{\varepsilon}\right)_{z}\right)^{2} d s d y d z \leq C \varepsilon^{2}
$$

We now require a generalization of Proposition 2.1. To this end, consider a node $P_{l}$ such that $M_{l}$ arcs of the graph $\Gamma$ meet there. For ease of notation, let us assume we have perhaps relabeled the arcs so that the collection meeting $P_{l}$ is given by parametrizations $\left\{\gamma_{k}\right\}, k=1, \ldots, M_{l}$. Without loss of generality, assume $P_{l}=\gamma_{k}(0)$ for each $k$ and denote by $D_{k}^{\varepsilon}$ the disc

$$
\left\{x: x=\gamma_{k}\left(\varepsilon^{1+p}\right)+y \varepsilon^{1+p} \mathbf{n}_{k}\left(\varepsilon^{1+p}\right)+z \varepsilon^{1+p} \mathbf{b}_{k}\left(\varepsilon^{1+p}\right) \text { for } y^{2}+z^{2}<1\right\} .
$$

(If $P_{l}=\gamma_{k}\left(L_{k}\right)$ one would simply replace $\varepsilon^{1+p}$ by $L_{k}-\varepsilon^{1+p}$ in the definition of $D_{k}^{\varepsilon}$.) Then we can establish:

3.4 Proposition. There exists a subsequence $\left\{\varepsilon_{j}\right\} \rightarrow 0$ such that for each $l \in$ $\{1, \ldots, M\}$ there is a number $\alpha_{l}^{0}$, independent of $k$, satisfying the condition

$$
\lim _{\varepsilon_{j} \rightarrow 0} f_{D_{k}^{\varepsilon_{j}}} \psi^{\varepsilon_{j}} d S=\alpha_{l}^{0} \quad \text { for } k=1, \ldots, M_{l} .
$$

Proof. In light of (3.12), as in the proof of Proposition 2.1 we may apply Lemma 2.3 to obtain the desired conclusion.

With Proposition 3.4 in hand, we now focus on the $k^{\text {th }}$ branch $\Gamma_{k}$ of the graph, whose two endpoints we denote by $P_{l_{1}}$ and $P_{l_{2}}$. We recall that we allow $P_{l_{1}}=P_{l_{2}}$ in the case of a closed arc. Now, as before, we take integral averages over cross-sections of $\mathcal{C}_{k}$ in defining $\bar{\Psi}_{k}^{\varepsilon}:\left[\varepsilon^{1+p}, L_{k}-\varepsilon^{1+p}\right] \rightarrow \mathbb{C}$ via

$$
\bar{\Psi}_{k}^{\varepsilon}(s)=\frac{1}{\pi} \int_{\left\{y^{2}+z^{2}<1\right\}} \Psi_{k}^{\varepsilon}(s, y, z) d y d z .
$$

Finally, we extend the definition so that $\bar{\Psi}_{k}^{\varepsilon}(s) \equiv \bar{\Psi}_{k}^{\varepsilon}\left(\varepsilon^{1+p}\right)$ for all $s<\varepsilon^{1+p}$ and $\bar{\Psi}_{k}^{\varepsilon}(s) \equiv \bar{\Psi}_{k}^{\varepsilon}\left(L_{k}-\varepsilon^{1+p}\right)$ for all $s>L_{k}-\varepsilon^{1+p}$.

Now we are ready to state the analogue of the compactness result Proposition 2.4 in the setting of constricted networks for the Ginzburg-Landau functional: 
3.5 Proposition. There exists a subsequence $\left\{\varepsilon_{j}\right\} \rightarrow 0$ and a function $\Psi^{0} \in$ $H^{1}\left(\Gamma \backslash\left\{P_{1} \cup \ldots \cup P_{M}\right\}\right)$ such that for any $k \in\{1, \ldots, N\}$ and for any $\delta>0$ one has

$$
\bar{\Psi}_{k}^{\varepsilon_{j}} \rightarrow \Psi_{k}^{0} \quad \text { weakly in } H^{1} \text { for } \quad \delta \leq s \leq L_{k}-\delta
$$

and

$$
\bar{\Psi}_{k}^{\varepsilon_{j}} \rightarrow \Psi_{k}^{0} \quad \text { on } \quad \delta \leq s \leq L_{k}-\delta
$$

weakly in $H^{1}$ as well as uniformly as $\varepsilon_{j} \rightarrow 0$. Here we have denoted $\Psi_{k}^{0}:=$ $\Psi^{0} \circ \gamma_{k}$.

Furthermore, we have the convergences

$$
\bar{\Psi}_{k}^{\varepsilon_{j}} \rightarrow\left\{\begin{array}{cc}
\alpha_{0}^{l_{1}} & \text { for } s<0 \\
\Psi_{k}^{0} & \text { for } 0<s<L_{k} \\
\alpha_{0}^{l_{2}} & \text { for } s>L_{k}
\end{array}\right.
$$

in $L^{1}\left(\left(-1, L_{k}+1\right)\right)$ and

$$
\begin{aligned}
& \left(\bar{\Psi}_{k}^{\varepsilon_{j}}\right)^{\prime} \stackrel{*}{\rightarrow} \\
& \left(\Psi_{k}^{0}\right)^{\prime}\left\llcorner\left\{0<s<L_{k}\right\}+\left(\Psi_{k}^{0}(0)-\alpha_{0}^{l_{1}}\right) \delta_{\{s=0\}}+\left(\alpha_{0}^{l_{2}}-\Psi_{k}^{0}\left(L_{k}\right)\right) \delta_{\left\{s=L_{k}\right\}},\right.
\end{aligned}
$$

weakly as measures, where we suppose the endpoints of the curve $\Gamma_{k}$ are $P_{l_{1}}$ and $P_{l_{2}}$.

Proof. The proof is very similar to that of Proposition 2.4 except that the inequalities (2.6) and (2.8) are replaced by (3.19). We will comment only on this distinction and leave out the rest of the details. Note that from (3.19) one obtains

$\int_{\mathcal{C}_{k}}\left|\left(\frac{1}{\zeta}\left(\Psi_{k}^{\varepsilon}\right)_{s}-i \mathcal{A}^{\varepsilon, T} \Psi_{k}^{\varepsilon}\right)-\left(\frac{\delta}{\zeta g_{k}^{\varepsilon}}\left(\Psi_{k}^{\varepsilon}\right)_{y}+\frac{\theta}{\zeta g_{k}^{\varepsilon}}\left(\Psi_{k}^{\varepsilon}\right)_{z}\right)\right|^{2} \frac{\left(g_{k}^{\varepsilon}\right)^{2} \zeta}{\varepsilon^{2}} d s d y d z \leq C_{1}$.

Since $\zeta \geq \frac{1}{2}$ while

$$
|\delta|+|\theta| \leq C \varepsilon^{1-p}
$$

(cf. (3.16))-(3.17)), we may invoke (3.14) and (3.21) to conclude that

$$
\int_{\mathfrak{C}_{k}} a_{k}^{\varepsilon}(s)\left|\left(\Psi_{k}^{\varepsilon}\right)_{s}\right|^{2} d s d y d z \leq C
$$

for some constant $C$, where we have defined

$$
a_{k}^{\varepsilon}(s):=\frac{g_{k}^{\varepsilon}(s)^{2}}{\varepsilon^{2}} .
$$


Note that (3.28), along with (3.21), in particular yield a uniform $H^{1}$-bound on the sequence $\left\{\Psi_{k}^{\varepsilon}\right\}$ restricted to the set $\left\{(s, y, z): \delta \leq s \leq L_{k}-\delta, y^{2}+z^{2}<\right.$ $1\}$ for any $\delta>0$. This establishes (3.23) Furthermore, we have obtained the direct analogue of (2.24) and the remainder of the proof follows that of Proposition 2.4 exactly with the use of Proposition 3.4 replacing that of Proposition 2.1.

Now we can state the main result of this section, asserting that the limit of minimizers of the Ginzburg-Landau energy necessarily solves a variational problem posed on the graph $\Gamma$ :

3.6 Theorem. The function $\Psi^{0}$ arising as a subsequential limit of minimizers of the Ginzburg-Landau energy in Proposition 3.5 along with the $M$ complex numbers $\alpha_{0}^{1}, \ldots, \alpha_{0}^{M}$ arising in Proposition 3.4 together solve the problem

$$
\begin{aligned}
& \inf _{\Psi \in H^{1}\left(\Gamma \backslash\left(\cup P_{l}\right)\right), \alpha_{1}, . ., \alpha_{M} \in \mathbb{C}} \sum_{k=1}^{N}\left[\int_{\Gamma_{k}}\left|\Psi_{k}^{\prime}-i \mathcal{A}_{k}^{e, T} \Psi_{k}\right|^{2}+\frac{\tilde{\nu}}{2}\left(\left|\Psi_{k}\right|^{2}-\mu^{2}\right)^{2} d s\right] \\
& \left.+b \sum_{l=1}^{M} \sum_{l^{\prime}=1}^{M_{l}}\left|\Psi_{k_{l^{\prime}}}\left(P_{l}\right)-\alpha_{l}\right|^{2}\right\},
\end{aligned}
$$

where $\Psi_{k}:=\Psi \circ \gamma_{k}, \mathcal{A}_{k}^{e, T}$ denotes the component of the applied potential $\mathbf{A}^{e}$ tangent to $\Gamma_{k}$ evaluated along $\Gamma_{k}$, that is,

$$
\mathcal{A}_{k}^{e, T}(s):=\mathbf{A}^{e}\left(\gamma_{k}(s)\right) \cdot \gamma_{k}^{\prime}(s)
$$

and in the last sum, $k_{l^{\prime}}$ ranges only over those curves $\Gamma_{k_{l^{\prime}}}$ having $P_{l}$ as an endpoint.

3.7 Remark. Setting the first variation of the limiting energy to zero, one finds that $\Psi^{0}$ satisfies the Euler-Lagrange equation

$$
\left(\frac{d}{d s}-i \mathcal{A}_{k}^{e, T}\right)^{2} \Psi_{k}^{0}=\left(1-\left|\Psi_{k}^{0}\right|^{2}\right) \Psi_{k}^{0} \quad \text { on each } \Gamma_{k}
$$

along with $M_{l}$ natural boundary conditions at each point $P_{l}$ :

$$
\left(\frac{d}{d s}-i A_{k_{l^{\prime}}}^{e, T}\right) \Psi_{k_{l^{\prime}}}^{0}(0)=b\left(\Psi_{k_{l^{\prime}}}^{0}(0)-\frac{1}{M_{l}} \sum_{j=1}^{M_{l}} \Psi_{k_{j}}^{0}(0)\right)
$$


for $l^{\prime}=1, \ldots, M_{l}$. In writing (3.33) we adopt the convention that $\gamma_{k_{l^{\prime}}}(0)=P_{l}$ with arclength $s$ being measured starting from $P_{l}$. In the case of a closed curve where $\gamma_{k_{l^{\prime}}}(0)=\gamma_{k_{l^{\prime}}}\left(L_{k}\right)$ we would write the corresponding term in the sum using $\Psi_{k_{l^{\prime}}}^{0}\left(L_{k}\right)$ on the right and replacing $\left(\frac{d}{d s}-i A_{k_{l^{\prime}}}^{e, T}\right) \Psi_{k_{l^{\prime}}}^{0}(0)$ with $\left(-\frac{d}{d s}-i A_{k_{l^{\prime}}}^{e, T}\right) \Psi_{k_{l^{\prime}}}^{0}\left(L_{k}\right)$ on the left.

3.8 Remark. Summing over $l^{\prime}$ in (3.33) we obtain a generalization of the "zero-flux" type condition (2.40) obtained in Section 2:

$$
\sum_{k_{l^{\prime}}=1}^{M_{l}}\left(\frac{d}{d s}-i A_{k_{l^{\prime}}}^{e, T}\right) \Psi_{k_{l^{\prime}}}^{0}(0)=0
$$

3.9 Remark. In a one-dimensional Ginzburg-Landau model, the supercurrent along a curve $\Gamma_{k_{l}}$ is defined as

$$
J_{k_{l}}:=\operatorname{Im}\left[\left(\Psi_{k_{l}}^{0}\right)^{*}\left(\frac{d}{d s}-i \mathcal{A}_{k_{l}}^{e, T}\right) \Psi_{k_{l}}^{0}\right]
$$

and consideration of the imaginary part of (3.32) leads to the conclusion that the current is constant on each branch of $\Gamma$. We can identify this constant if we express $\Psi_{k_{l^{\prime}}}^{0}$ and $\Psi_{k_{j}}^{0}$ in polar form: $\Psi_{k_{l^{\prime}}}^{0}=\rho_{k_{l^{\prime}}} e^{i \phi_{k_{l^{\prime}}}}$ and $\Psi_{k_{j}}^{0}=\rho_{k_{j}} e^{i \phi_{k_{j}}}$. Simply multiply (3.33) by $\left(\Psi_{k^{\prime}}^{0}\right)^{*}$ and a short calculation yields the following generalized Josephson condition:

$$
J_{k_{l^{\prime}}}=\frac{b}{M_{l}} \sum_{j=1}^{M_{l}} \rho_{k_{j}} \rho_{k_{l^{\prime}}} \sin \left(\phi_{k_{j}}-\phi_{k_{l^{\prime}}}\right) \quad \text { for } l^{\prime}=1,2, \ldots, M_{l} .
$$

Note that there will be $M_{l}-1$ terms in the sum (3.34), since the term corresponding to $k_{j}=k_{l^{\prime}}$ vanishes. Also, due to the oddness of the sine function, note that the total current coming into a node on the graph will be zero, that is

$$
\sum_{l^{\prime}=1}^{M_{l}} J_{k_{l^{\prime}}}=0
$$

Proof. Since the parameters $\tilde{\nu}$ and $\mu$ play no significant role in the argument, we will set both equal to one in the proof. The proof mirrors that of Theorem 2.5. The identification of $\Psi^{0}$ and $\left(\alpha_{0}^{1}, \ldots, \alpha_{0}^{M}\right)$ as minimizers of (3.30) will result from establishing the following two claims. First we will show that

$$
\begin{aligned}
& \liminf _{\varepsilon_{j} \rightarrow 0} G_{\varepsilon_{j}}\left(\psi^{\varepsilon_{j}}, \mathbf{A}^{\varepsilon_{j}}\right) \geq \\
& \pi\left\{\sum_{k=1}^{N}\left[\int_{\Gamma_{k}}\left|\left(\Psi_{k}^{0}\right)^{\prime}-i \mathcal{A}_{k}^{e, T} \Psi_{k}^{0}\right|^{2}+\frac{1}{2}\left(\left|\Psi_{k}^{0}\right|^{2}-1\right)^{2} d s\right]+b \sum_{l=1}^{M} \sum_{l^{\prime}=1}^{M_{l}}\left|\Psi_{k^{\prime}}^{0}\left(P_{l}\right)-\alpha_{0}^{l}\right|^{2}\right\} .
\end{aligned}
$$


Then we will show that for any $\Psi \in H^{1}\left(\Gamma \backslash \cup\left\{P_{l}\right\}\right)$ and any numbers $\alpha_{1}, \ldots, \alpha_{M} \in \mathbb{C}$, there exists a sequence $\left\{\Phi^{\varepsilon}\right\}$ with $\Phi^{\varepsilon} \in H^{1}\left(\Omega_{\varepsilon}\right)$ such that

$$
\begin{aligned}
& \lim _{\varepsilon \rightarrow 0} G_{\varepsilon}\left(\Phi^{\varepsilon}, \mathbf{A}^{e}\right)= \\
& \pi\left\{\sum_{k=1}^{N}\left[\int_{\Gamma_{k}}\left|\Psi_{k}^{\prime}-i \mathcal{A}_{k}^{e, T} \Psi_{k}\right|^{2}+\frac{1}{2}\left(\left|\Psi_{k}\right|^{2}-1\right)^{2} d s\right]+b \sum_{l=1}^{M} \sum_{l^{\prime}=1}^{M_{l}}\left|\Psi_{k_{l^{\prime}}}\left(P_{l}\right)-\alpha_{l}\right|^{2}\right\} .
\end{aligned}
$$

Conditions (3.35), (3.36) and the fact that the pair $\left(\psi^{\varepsilon}, \mathbf{A}^{\varepsilon}\right)$ minimizes $G_{\varepsilon}$ then yield the desired result.

Proof of Claim (3.35). Invoking (3.18), (3.19), (3.21) and the fact that $\zeta \rightarrow 1$ as $\varepsilon \rightarrow 0$, it is straight-forward to check that

$$
\begin{aligned}
& \liminf _{\varepsilon_{j} \rightarrow 0} G_{\varepsilon_{j}}\left(\psi^{\varepsilon_{j}}, \mathbf{A}^{\varepsilon_{j}}\right) \geq \\
& \liminf _{\varepsilon_{j} \rightarrow 0} \sum_{k=1}^{N} \int_{\varepsilon_{j}^{1+p}}^{L_{k}-\varepsilon_{j}^{1+p}} \int_{\left\{y^{2}+z^{2}<1\right\}} \\
& \left|\left(\frac{1}{\zeta}\left(\Psi_{k}^{\varepsilon_{j}}\right)_{s}-i \mathcal{A}^{\varepsilon_{j}, T} \Psi_{k}^{\varepsilon_{j}}\right)-\left(\frac{\delta}{\zeta g_{k}^{\varepsilon_{j}}}\left(\Psi_{k}^{\varepsilon_{j}}\right)_{y}+\frac{\theta}{\zeta g_{k}^{\varepsilon_{j}}}\left(\Psi_{k}^{\varepsilon_{j}}\right)_{z}\right)\right|^{2} \frac{\left(g_{k}^{\varepsilon_{j}}\right)^{2} \zeta}{\varepsilon_{j}^{2}} d y d z d s \\
& +\liminf _{\varepsilon_{j} \rightarrow 0} \sum_{k=1}^{N} \frac{1}{2} \int_{\varepsilon_{j}^{p}}^{L_{k}-\varepsilon_{j}^{p}} \int_{\left\{y^{2}+z^{2}<1\right\}}\left(\left|\Psi_{k}^{\varepsilon_{j}}\right|^{2}-1\right)^{2} \zeta d y d z d s \geq \\
& \liminf _{\varepsilon_{j} \rightarrow 0} \sum_{k=1}^{N} \int_{\varepsilon_{j}^{1+p}}^{L_{k}-\varepsilon_{j}^{1+p}} \int_{\left\{y^{2}+z^{2}<1\right\}} a_{k}^{\varepsilon_{j}}(s)\left|\left(\Psi_{k}^{\varepsilon_{j}}\right)_{s}-i \mathcal{A}_{1}^{\varepsilon_{j}} \Psi_{k}^{\varepsilon_{j}}\right|^{2} d y d z d s \\
& +\liminf _{\varepsilon_{j} \rightarrow 0} \sum_{k=1}^{N} \frac{1}{2} \int_{\varepsilon_{j}^{p}}^{L_{k}-\varepsilon_{j}^{p}} \int_{\left\{y^{2}+z^{2}<1\right\}}\left(\left|\Psi_{k}^{\varepsilon_{j}}\right|^{2}-1\right)^{2} d y d z d s .
\end{aligned}
$$

Observe next that (3.23) implies strong $L^{4}$-convergence of $\Psi_{k}^{\varepsilon_{j}}$ to $\Psi_{k}^{0}$ along a subsequence and that (3.14) in particular implies that $\mathcal{A}^{\varepsilon_{j}, T} \rightarrow \mathcal{A}_{k}^{e, T}$ in $L^{\infty}$. Applying these facts to the last limit above, one finds that

$$
\begin{aligned}
& \liminf _{\varepsilon_{j} \rightarrow 0} G_{\varepsilon_{j}}\left(\psi^{\varepsilon_{j}}, \mathbf{A}^{\varepsilon_{j}}\right) \geq \\
& \liminf _{\varepsilon_{j} \rightarrow 0} \sum_{k=1}^{N} \int_{\varepsilon_{j}^{1+p}}^{L_{k}-\varepsilon_{j}^{1+p}} \int_{\left\{y^{2}+z^{2}<1\right\}} a_{k}^{\varepsilon}(s)\left|\left(\Psi_{k}^{\varepsilon_{j}}\right)_{s}-i \mathcal{A}_{k}^{e, T} \Psi_{k}^{\varepsilon_{j}}\right|^{2} d y d z d s \\
& +\pi \sum_{k=1}^{N} \frac{1}{2} \int_{0}^{L_{k}}\left(\left|\Psi_{k}^{0}\right|^{2}-1\right)^{2} d s .
\end{aligned}
$$

Let us now fix any $k \in\{1, \ldots, N\}$ and suppose the curve $\Gamma_{k}$ has endpoints $P_{l_{1}}$ 
and $P_{l_{2}}$. Expanding the square $\left|\left(\Psi_{k}^{\varepsilon_{j}}\right)_{s}-i \mathcal{A}_{k}^{e, T} \Psi_{k}^{\varepsilon_{j}}\right|^{2}$, we can invoke Proposition 3.5 to obtain

$$
\begin{aligned}
& \lim _{\varepsilon_{j} \rightarrow 0} \int_{\varepsilon_{j}^{1+p}}^{L_{k}-\varepsilon_{j}^{1+p}} \int_{\left\{y^{2}+z^{2}<1\right\}} i\left(\left(\Psi_{k}^{\varepsilon_{j}}\right)^{*}\left(\Psi_{k}^{\varepsilon_{j}}\right)_{s}-\left(\Psi_{k}^{\varepsilon_{j}}\right)\left(\Psi_{k}^{\varepsilon_{j}}\right)_{s}^{*}\right) \mathcal{A}_{k}^{e, T}+\left|\mathcal{A}_{k}^{e, T} \Psi_{k}^{\varepsilon_{j}}\right|^{2} d y d z d s \\
& =\pi \int_{0}^{L_{k}} i\left(\left(\Psi_{k}^{0}\right)^{*}\left(\Psi_{k}^{0}\right)_{s}-\left(\Psi_{k}^{0}\right)\left(\Psi_{k}^{0}\right)_{s}^{*}\right) \mathcal{A}_{k}^{e, T}+\left|\mathcal{A}^{e, T} \Psi_{k}^{0}\right|^{2} d s
\end{aligned}
$$

Now we appeal to Theorem 3.1 of [3] (see also [2]) to handle the limit of the integral of the remaining term

$$
a_{k}^{\varepsilon_{j}}(s)\left|\left(\Psi_{k}^{\varepsilon_{j}}\right)_{s}\right|^{2}
$$

as was done in the proof of claim (2.30) in Section 2. Specifically, we apply the Cauchy-Schwartz inequality followed by (2.21)-(2.27), (3.2), (3.24), (3.26) to see that

$$
\begin{aligned}
& \liminf _{\varepsilon_{j} \rightarrow 0} \int_{\varepsilon_{j}^{1+p}}^{L_{k}-\varepsilon_{j}^{1+p}} \int_{\left\{y^{2}+z^{2}<1\right\}} a_{k}^{\varepsilon_{j}}(s)\left|\left(\Psi_{k}^{\varepsilon_{j}}\right)_{s}\right|^{2} d y d z d s \geq \\
& \pi \liminf _{\varepsilon_{j} \rightarrow 0} \int_{\varepsilon_{j}^{1+p}}^{L_{k}-\varepsilon_{j}^{1+p}} a_{k}^{\varepsilon_{j}}(s)\left|\left(\bar{\Psi}_{k}^{\varepsilon_{j}}\right)^{\prime}\right|^{2} d s=\pi \liminf _{\varepsilon_{j} \rightarrow 0} \int_{-1}^{L_{k}+1} a_{k}^{\varepsilon_{j}}(s)\left|\left(\bar{\Psi}_{k}^{\varepsilon_{j}}\right)^{\prime}\right|^{2} d s \geq \\
& \pi\left\{\int_{0}^{L_{k}}\left|\left(\Psi_{k}^{0}\right)^{\prime}\right|^{2} d s+b\left|\Psi_{k}^{0}(0)-\alpha_{0}^{l_{1}}\right|^{2}+b\left|\Psi_{k}^{0}\left(L_{k}\right)-\alpha_{0}^{l_{2}}\right|^{2}\right\} .
\end{aligned}
$$

Claim (3.35) follows from (3.37)-(3.39).

Proof of Claim (3.36). Fix any $\Psi \in H^{1}\left(\Gamma \backslash \cup\left\{P_{l}\right\}\right)$ and any numbers $\alpha_{1}, \ldots, \alpha_{M} \in \mathbb{C}$. As has been our convention throughout, we write $\Psi_{k}$ for the composition $\Psi \circ \gamma_{k}$. In order to define the desired sequence $\left\{\Phi^{\varepsilon}\right\}$, we will need to make a few definitions. In addition to the previously defined tangential component of the applied potential $\mathcal{A}_{k}^{e, T}$, for each $k$ we now introduce

$$
\mathcal{A}_{k}^{e, n}(s):=\mathbf{A}^{e}\left(\gamma_{k}(s)\right) \cdot \mathbf{n}_{k}(s) \quad \text { and } \quad \mathcal{A}_{k}^{e, b}(s):=\mathbf{A}^{e}\left(\gamma_{k}(s)\right) \cdot \mathbf{b}_{k}(s)
$$

as the normal and binormal components evaluated along $\Gamma_{k}$. We also define

$$
\lambda_{k}^{\varepsilon}(s):=\frac{1}{a_{k}^{\varepsilon}(s)}-1 \quad \text { and } \quad \beta_{k}^{\varepsilon}:=\int_{\varepsilon^{1+p}}^{L_{k} / 2} \lambda_{k}^{\varepsilon}(s) d s .
$$

Note that

$$
\lambda_{k}^{\varepsilon}(s) \equiv 0 \quad \text { for } \quad \varepsilon^{p}<s<L_{k}-\varepsilon^{p},
$$

since $a_{k}^{\varepsilon}(s) \equiv 1$ on that $s$ interval, while from (3.2) and (2.26) we have that

$$
\beta_{k}^{\varepsilon}=\int_{L_{k} / 2}^{L_{k}-\varepsilon^{1+p}} \lambda_{k}^{\varepsilon}(s) d s \quad \text { and } \quad \beta_{k}^{\varepsilon} \rightarrow \frac{1}{b} \quad \text { as } \varepsilon \rightarrow 0 .
$$


With these definition in hand, let us consider any $k \in\{1, \ldots, N\}$ with the curve $\Gamma_{k}$ having endpoints $P_{l_{1}}$ and $P_{l_{2}}$. On the interval $\varepsilon^{1+p}<s<L_{k}-\varepsilon^{1+p}$ we first define a sequence of functions $\tilde{\Phi}_{k}^{\varepsilon}$ depending only on $s$ by the formula $\tilde{\Phi}_{k}^{\varepsilon}(s)=\left\{\begin{array}{c}\Psi_{k}(s)-\frac{1}{\beta_{k}^{\varepsilon}}\left(\Psi_{k}\left(\varepsilon^{1+p}\right)-\alpha_{l_{1}}\right) \int_{s}^{L_{k} / 2} \lambda_{k}^{\varepsilon}\left(s^{\prime}\right) d s^{\prime} \quad \text { for } \quad \varepsilon^{1+p}<s \leq L_{k} / 2, \\ \Psi_{k}(s)-\frac{1}{\beta_{k}^{\varepsilon}}\left(\Psi_{k}\left(L_{k}-\varepsilon^{1+p}\right)-\alpha_{l_{2}}\right) \int_{L_{k} / 2}^{s} \lambda_{k}^{\varepsilon}\left(s^{\prime}\right) d s^{\prime} \quad \text { for } \quad L_{k} / 2<s<L_{k}-\varepsilon^{1+p} .\end{array}\right.$

Then on the set

$$
\Omega_{\varepsilon, k}:=T_{k}^{\varepsilon}\left(\left\{(s, y, z): \varepsilon^{1+p}<s<L_{k}-\varepsilon^{1+p}, y^{2}+z^{2}<1\right\}\right)
$$

(cf. (3.3)), we define our sequence $\left\{\Phi^{\varepsilon}\right\}$ as a function of $s, y$ and $z$ via

$$
\Phi_{k}^{\varepsilon}(s, y, z):=\tilde{\Phi}_{k}^{\varepsilon}(s) e^{i g_{k}^{\varepsilon}(s)\left[\mathcal{A}_{k}^{e, n}(s) y+\mathcal{A}_{k}^{e, b}(s) z\right] \eta_{k}^{\varepsilon}(s)}
$$

where $\eta_{k}^{\varepsilon}:\left[\varepsilon^{1+p}, L_{k}-\varepsilon^{1+p}\right] \rightarrow \mathbb{R}^{1}$ is the cut-off function given by $\eta_{k}^{\varepsilon}(s)=1$ for $2 \varepsilon^{1+p}<s<L_{k}-2 \varepsilon^{1+p}, \eta_{k}^{\varepsilon}\left(\varepsilon^{1+p}\right)=0=\eta_{k}^{\varepsilon}\left(L_{k}-\varepsilon^{1+p}\right)$ and $\eta_{k}^{\varepsilon}$ linear on the intervals $\varepsilon^{1+p}<s<2 \varepsilon^{1+p}$ and $L_{k}-2 \varepsilon^{1+p}<s<L_{k}-\varepsilon^{1+p}$. The reason for the additional phase exhibited in (3.43) will become apparent in (3.47) below.

Since $\Phi^{\varepsilon}$ should be a function defined on $\Omega_{\varepsilon}$, strictly speaking, we really mean that on the $k^{\text {th }}$ branch of $\Omega_{\varepsilon}, \Phi^{\varepsilon}=\Phi_{k}^{\varepsilon}\left(\left(T_{k}^{\varepsilon}\right)^{-1}\left(x_{1}, x_{2}, x_{3}\right)\right)$ when we write $\Phi_{k}^{\varepsilon}(s, y, z)$ but we will not make this distinction and we trust that this will not lead to confusion.

To complete the definition of $\Phi^{\varepsilon}$ on $\Omega_{\varepsilon}$ we set $\Phi^{\varepsilon} \equiv \alpha_{l}$ on the component of $\Omega_{\varepsilon} \backslash\left(\cup_{k} \Omega_{\varepsilon, k}\right)$ that contains the point $P_{l}$.

Now we are ready to compute $\lim _{\varepsilon \rightarrow 0} G_{\varepsilon}\left(\Phi^{\varepsilon}, \mathbf{A}^{e}\right)$. Let us first remark that since $\Phi^{\varepsilon}$ is constant on $\Omega_{\varepsilon} \backslash\left(\cup_{k} \Omega_{\varepsilon, k}\right)$, a set whose measure is $\mathcal{O}\left(\varepsilon^{3+3 p}\right)$, the contribution to the total energy from integration over this set will be $\mathcal{O}\left(\varepsilon^{1+3 p}\right)$ and so it can be ignored in the limit.

We next compute the limit of the potential term in the Ginzburg-Landau energy. Since (3.40) implies that $\left|\Phi_{k}^{\varepsilon}(s, y, z)\right|=\left|\Psi_{k}(s)\right|$ for $\varepsilon^{p}<s<L_{k}-\varepsilon^{p}$ we can easily check that

$$
\frac{1}{\varepsilon^{2}} \int_{\Omega_{\varepsilon, k}}\left(\left|\Phi^{\varepsilon}\right|^{2}-1\right)^{2} d x=\pi \int_{\varepsilon^{p}}^{L_{k}-\varepsilon^{1+p}}\left(\left|\Psi_{k}\right|^{2}-1\right)^{2} d s+\mathcal{O}\left(\varepsilon^{p}\right)
$$

and so

$$
\lim _{\varepsilon \rightarrow 0} \frac{1}{\varepsilon^{2}} \int_{\Omega_{\varepsilon, k}}\left(\left|\Phi^{\varepsilon}\right|^{2}-1\right)^{2} d x=\pi \int_{0}^{L_{k}}\left(\left|\Psi_{k}\right|^{2}-1\right)^{2} d s
$$


Now we turn to the limit of the term

$$
\frac{1}{\varepsilon^{2}} \int_{\Omega_{\varepsilon, k}}\left|\left(\nabla-i \mathbf{A}^{e}\right) \Phi^{\varepsilon}\right|^{2} d x
$$

In a calculation analogous to (3.19), we find

$$
\begin{aligned}
& \lim _{\varepsilon \rightarrow 0} \frac{1}{\varepsilon^{2}} \int_{\Omega_{\varepsilon, k}}\left|\left(\nabla-i \mathbf{A}^{e}\right) \Phi^{\varepsilon}\right|^{2} d x= \\
& \lim _{\varepsilon \rightarrow 0} \int_{\varepsilon^{1+p}}^{L_{k}-\varepsilon^{1+p}} \int_{\left\{y^{2}+z^{2}<1\right\}} \\
& \left|\left(\frac{1}{\zeta}\left(\Phi_{k}^{\varepsilon}\right)_{s}-i \mathcal{A}^{e, T} \Phi_{k}^{\varepsilon}\right)-\left(\frac{\delta}{\zeta g_{k}^{\varepsilon}}\left(\Phi_{k}^{\varepsilon}\right)_{y}+\frac{\theta}{\zeta g_{k}^{\varepsilon}}\left(\Phi_{k}^{\varepsilon}\right)_{z}\right)\right|^{2} \frac{\left(g_{k}^{\varepsilon}\right)^{2} \zeta}{\varepsilon^{2}} d y d z d s .
\end{aligned}
$$

Notice that there is no integral above corresponding to the last one in (3.19) since

$$
\left(\Phi_{k}^{\varepsilon}\right)_{y}-i g_{k}^{\varepsilon} \mathbf{A}^{e}\left(T_{k}^{\varepsilon}\right) \cdot \mathbf{n}_{k} \Phi_{k}^{\varepsilon}=i g_{k}^{\varepsilon} \Phi_{k}^{\varepsilon}\left[\mathbf{A}^{e}\left(T_{k}^{\varepsilon}(s, 0,0)-\mathbf{A}^{e}\left(T_{k}^{\varepsilon}(s, y, z)\right) \cdot \mathbf{n}_{k}(s)\right)\right]
$$

so that

$$
\begin{aligned}
& \int_{\varepsilon^{p}}^{L_{k}-\varepsilon^{p}} \int_{\left\{y^{2}+z^{2}<1\right\}}\left|\left(\Phi_{k}^{\varepsilon}\right)_{y}-i g_{k}^{\varepsilon} \mathbf{A}^{e}\left(T_{k}^{\varepsilon}\right) \cdot \mathbf{n}_{k} \Phi_{k}^{\varepsilon}\right|^{2} \frac{\zeta}{\varepsilon^{2}} d y d z d s= \\
& \mathcal{O}\left(\frac{\left(g_{k}^{\varepsilon}\right)^{2}}{\varepsilon^{2}}\right) \cdot o(1) \rightarrow 0,
\end{aligned}
$$

with a similar estimate holding for the difference between the $z$-derivative of $\Phi_{k}^{\varepsilon}$ and the $\mathbf{b}_{k}$ component of $\mathbf{A}^{e}$.

Returning then to (3.46), we observe through (3.27) and (3.43) that

$$
\left|\left(\frac{\delta}{\zeta g_{k}^{\varepsilon}}\left(\Phi_{k}^{\varepsilon}\right)_{y}+\frac{\theta}{\zeta g_{k}^{\varepsilon}}\left(\Phi_{k}^{\varepsilon}\right)_{z}\right)\right|^{2} \frac{\left(g_{k}^{\varepsilon}\right)^{2} \zeta}{\varepsilon^{2}}=\mathcal{O}\left(\frac{\left(g_{k}^{\varepsilon}(s)\right)^{2}}{\varepsilon^{2 p}}\right)=\mathcal{O}\left(\varepsilon^{2-2 p}\right) \rightarrow 0,
$$

This fact, along with $\zeta \rightarrow 1$, allows us to conclude that

$$
\begin{aligned}
& \lim _{\varepsilon \rightarrow 0} \frac{1}{\varepsilon^{2}} \int_{\Omega_{\varepsilon, k}}\left|\left(\nabla-i \mathbf{A}^{e}\right) \Phi^{\varepsilon}\right|^{2} d x= \\
& \lim _{\varepsilon \rightarrow 0} \int_{\varepsilon^{1+p}}^{L_{k}-\varepsilon^{1+p}} \int_{\left\{y^{2}+z^{2}<1\right\}} a_{k}^{\varepsilon}(s)\left|\left(\Phi_{k}^{\varepsilon}\right)_{s}-i \mathcal{A}^{e, T} \Phi_{k}^{\varepsilon}\right|^{2} d y d z d s .
\end{aligned}
$$

Then we note in light of (3.43) that

$$
\begin{aligned}
& \lim _{\varepsilon \rightarrow 0} \int_{\varepsilon^{1+p}}^{L_{k}-\varepsilon^{1+p}} \int_{\left\{y^{2}+z^{2}<1\right\}} a_{k}^{\varepsilon}(s)\left|\left(\Phi_{k}^{\varepsilon}\right)_{s}-i \mathcal{A}^{e, T} \Phi_{k}^{\varepsilon}\right|^{2} d y d z d s= \\
& \lim _{\varepsilon \rightarrow 0} \int_{\varepsilon^{1+p}}^{L_{k}-\varepsilon^{1+p}} \int_{\left\{y^{2}+z^{2}<1\right\}} a_{k}^{\varepsilon}(s)\left|\left(\tilde{\Phi}_{k}^{\varepsilon}\right)_{s}-i \mathcal{A}^{e, T} \tilde{\Phi}_{k}^{\varepsilon}\right|^{2} d y d z d s
\end{aligned}
$$


since

$$
\left(\Phi_{k}^{\varepsilon}\right)_{s}=\left[\left(\tilde{\Phi}_{k}^{\varepsilon}\right)_{s}+\tilde{\Phi}_{k}^{\varepsilon} \frac{d}{d s}\left\{i g_{k}^{\varepsilon}(s)\left[\mathcal{A}_{k}^{e, n}(s) y+\mathcal{A}_{k}^{e, b}(s) z\right] \eta_{k}^{\varepsilon}(s)\right\}\right] e^{i g_{k}^{\varepsilon}(s)\left[\mathcal{A}_{k}^{e, n}(s) y+\mathcal{A}_{k}^{e, b}(s) z\right] \eta_{k}^{\varepsilon}(s)} .
$$

Then using the definition of the cut-off function $\eta_{k}^{\varepsilon}$ one readily confirms that

$\int_{\varepsilon^{1+p}}^{L_{k}-\varepsilon^{1+p}} \int_{\left\{y^{2}+z^{2}<1\right\}} a_{k}^{\varepsilon}(s)\left|\frac{d}{d s}\left\{i g_{k}^{\varepsilon}(s)\left[\mathcal{A}_{k}^{e, n}(s) y+\mathcal{A}_{k}^{e, b}(s) z\right] \eta_{k}^{\varepsilon}(s)\right\}\right|^{2} d y d z d s \rightarrow 0$.

Invoking (3.40), we can then substitute the definition of $\tilde{\Phi}_{k}^{\varepsilon}$ into (3.51) to find

$$
\begin{aligned}
& \lim _{\varepsilon \rightarrow 0} \int_{\varepsilon^{1+p}}^{L_{k}-\varepsilon^{1+p}} \int_{\left\{y^{2}+z^{2}<1\right\}} a_{k}^{\varepsilon}(s)\left|\left(\tilde{\Phi}_{k}^{\varepsilon}\right)_{s}-i \mathcal{A}_{k}^{e, T} \tilde{\Phi}_{k}^{\varepsilon}\right|^{2} d y d z d s= \\
& \lim _{\varepsilon \rightarrow 0} \int_{\varepsilon^{p}}^{L_{k}-\varepsilon^{p}} \int_{\left\{y^{2}+z^{2}<1\right\}}\left|\left(\Psi_{k}\right)_{s}-i \mathcal{A}_{k}^{e, T} \Psi_{k}\right|^{2} d y d z d s+ \\
& \lim _{\varepsilon \rightarrow 0} \int_{\varepsilon^{1+p}}^{\varepsilon^{p}} \int_{\left\{y^{2}+z^{2}<1\right\}} a_{k}^{\varepsilon}(s)\left|\left(\Psi_{k}\right)_{s}\right|^{2} d y d z d s+ \\
& \lim _{\varepsilon \rightarrow 0} \int_{L_{k}-\varepsilon^{p}}^{L_{k}-\varepsilon^{1+p}} \int_{\left\{y^{2}+z^{2}<1\right\}} a_{k}^{\varepsilon}(s)\left|\left(\Psi_{k}\right)_{s}\right|^{2} d y d z d s,
\end{aligned}
$$

since

$$
\begin{aligned}
& \lim _{\varepsilon \rightarrow 0} \int_{\varepsilon^{1+p}}^{\varepsilon^{p}} \int_{\left\{y^{2}+z^{2}<1\right\}} a_{k}^{\varepsilon}(s)\left|\mathcal{A}_{k}^{e, T} \Psi_{k}\right|^{2} d y d z d s=0 \\
& =\lim _{\varepsilon \rightarrow 0} \int_{L_{k}-\varepsilon^{p}}^{L_{k}-\varepsilon^{1+p}} \int_{\left\{y^{2}+z^{2}<1\right\}} a_{k}^{\varepsilon}(s)\left|\mathcal{A}_{k}^{e, T} \Psi_{k}\right|^{2} d y d z d s .
\end{aligned}
$$

We conclude that

$$
\begin{aligned}
& \lim _{\varepsilon \rightarrow 0} \frac{1}{\varepsilon^{2}} \int_{\Omega_{\varepsilon, k}}\left|\left(\nabla-i \mathbf{A}^{e}\right) \Phi^{\varepsilon}\right|^{2} d x= \\
& \pi\left\{\int_{\Gamma_{k}}\left|\Psi_{k}^{\prime}-i \mathcal{A}_{k}^{e, T} \Psi_{k}\right|^{2}+\frac{1}{2}\left(1-\left|\Psi_{k}\right|^{2}\right)^{2} d s+b\left|\Psi_{k}(0)-\alpha_{l_{1}}\right|^{2}+b\left|\Psi_{k}\left(L_{k}\right)-\alpha_{l_{2}}\right|^{2}\right\}
\end{aligned}
$$

using the same type of calculation that concluded the proof of (2.31). Combining this with (3.44) and summing over $k$ we arrive at (3.36).

\section{Discussion}

We have characterized the asymptotic behavior of minimizers to two energies, the Dirichlet integral and the Ginzburg-Landau energy, taken over thin 
domains with constrictions. In both cases, the limit of these minimizers turns out to minimize a variational problem posed on the limiting graph. Certainly the most prominent feature of the limiting variational problem is that in general solutions will develop jump discontinuities at the nodes of the graph that mark the location of the constrictions. On an intuitive level, it is perhaps not surprising that large gradients might develop near the constrictions for the problems on the thickened graphs (i.e. when $\varepsilon>0$ ), but the subtlety here is, first of all, that the large gradients develop along the tapered interval $\varepsilon^{1+p}<s<\varepsilon^{p}$ where the domain radius $g^{\varepsilon}$ is linear rather than in an $\varepsilon^{1+p}$ neighborhood of the nodes. This is the thrust of Lemma 2.1 and Lemma 3.4. Secondly, the large gradient is not uniformly large along this interval of $s$-values. For instance, if instead of the construction (2.36), whose derivative grows as one nears the constriction, one were to try linking the value $\alpha$ near the node to a number $V_{k}(0)$ via a linear construction, one would find that the energy $\int a_{\varepsilon}\left|\left(V_{k}^{\varepsilon}\right)^{\prime}\right|^{2} d s$ blows up.

The approach is fairly robust in that the energies considered here can be replaced by more general elliptic integrands. Furthermore, the specific geometry of the constriction used here is not crucial. What is needed most crucially is that the cross-sectional radius $g^{\varepsilon}$ tapers as $s$ approaches zero, from a value $\varepsilon$ to something much smaller over an $s$-interval $0<s<s_{\varepsilon}$ with $s_{\varepsilon} \rightarrow 0$ in such a way that the condition

$$
C_{1}<\int_{0}^{s_{\varepsilon}} \frac{1}{a_{\varepsilon}} d s:=\int_{0}^{s_{\varepsilon}} \frac{\varepsilon^{2}}{\left(g^{\varepsilon}\right)^{2}} d s<C_{2}
$$

holds for positive constants $C_{1}$ and $C_{2}$ that are independent of $\varepsilon$. Perhaps the simplest alternative geometry for a constriction obeying this condition would be to take $g^{\varepsilon}$ as piecewise constant with $g^{\varepsilon}(s)=\varepsilon$ for $s>s_{\varepsilon}:=\varepsilon^{r}$ and $g^{\varepsilon}(s)=\varepsilon^{t}$ for $0 \leq s \leq \varepsilon^{r}$ where $r>0$ and $t=1+r / 2$. Formally, this geometry leads to the same type of $\Gamma$-limit as the one obtained in this paper, though we have not attempted to check it rigorously.

\section{References}

[1] A. Barone and G. Paterno, Physics and Applications of the Josephson Effect, Wiley, 1982.

[2] G. Bouchitté, Représentation int'egrale de fonctionnelles convexes sur un espace de mesures. II. Cas de l'épi-convergence, Ann. Univ. Ferrara. Sez. VII (N.S.), 33, 113-156, (1987). 
[3] G. Buttazzo and L. Freddi, Functionals defined on measures and applications to non-equi-uniformly elliptic problems, Ann. Mat. Pura App., 159, 133-149, (1991).

[4] E. Cabib, L. Freddi, A. Morassi and D. Percivale, Thin notched beams, J. Elasticity, 64, no. 2-3, 157-178, (2002).

[5] P.G de Gennes, Superconductivity of Metals and Alloys, Addison-Wesley (1989).

[6] Q. Du, M.D. Gunzburger and J.S. Peterson, Analysis and approximation of the Ginzburg-Landau model of superconductivity, SIAM Review 34, 45-81 (1992).

[7] L.C. Evans, Partial Differential Equations, Graduate Studies in Math. vol 19, A.M.S. (1998).

[8] S. Jimbo and P. Sternberg, Non-existence of Permanent Currents in Convex Planar Samples, SIAM J. Math. Anal. 33, no. 6, 1379-1392, (2002).

[9] B.D. Josephson, Possible new effects in superconducting tunneling, Phys. Rev. Lett., 1, 251-253, (1962).

[10] P. Kuchment and H. Zeng, Copnvergence of spectra of mesoscopic systems collapsing onto a graph, J. Math. Anal. Appl.,258, 671-700, (2001).

[11] P. Kuchment and H. Zeng, Asymptotics of spectra of Neumann Laplacians in thin domains, Cont. Math. 327, 199-213, (2003).

[12] K. Likharev, Superconducting weak links, Rev. Mod. Phys. 51, 101-159, (1979).

[13] J. Rubinstein and M. Schatzman, Variational problems in multiply connected thin strips II: The asymptotic limit of the Ginzburg Landau functional, Arch. Rat. Mech. Anal., 160, 309-324, (2001).

[14] J. Rubinstein and M. Schatzman, On mesoscopic superconducting samples, Proc. of the Third Int. Palestinian Conf., Math. and Educ., S. Elyadi et. al. eds., World Scientific, 253-263, (2002). 
[15] J. Rubinstein, M. Schatzman and P. Sternberg, Ginzburg-Landau model in thin loops with narrow constrictions, SIAM J. Appl. Math., 64, 21862204, (2004).

[16] J. Rubinstein, P. Sternberg and G. Wolansky, Transport in networks with constricted junctions, in preparation.

[17] W.P. Ziemer, Weakly Differentiable Functions, Springer-Verlag, (1989). 